\title{
All-sky photogrammetry techniques to georeference a cloud field
}

\author{
Pierre Crispel $^{1, \mathrm{a}}$ and Gregory Roberts ${ }^{1,2}$ \\ ${ }^{1}$ CNRM, Centre National de Recherches Météorologiques UMR 3589, Météo-France/CNRS, Toulouse, France \\ ${ }^{2}$ Scripps Institution of Oceanography, Center for Atmospheric Sciences and Physical Oceanography, \\ La Jolla, California, USA \\ anow at: Météo-France DSM/AERO, Toulouse, France
}

Correspondence: Pierre Crispel (pierre.crispel@ meteo.fr) and Gregory Roberts (greg.roberts@ meteo.fr)

Received: 20 June 2017 - Discussion started: 31 August 2017

Revised: 8 December 2017 - Accepted: 13 December 2017 - Published: 31 January 2018

\begin{abstract}
In this study, we present a novel method of identifying and geolocalizing cloud field elements from a portable all-sky camera stereo network based on the ground and oriented towards zenith. The methodology is mainly based on stereophotogrammetry which is a 3-D reconstruction technique based on triangulation from corresponding stereo pixels in rectified images. In cases where clouds are horizontally separated, identifying individual positions is performed with segmentation techniques based on hue filtering and contour detection algorithms. Macroscopic cloud field characteristics such as cloud layer base heights and velocity fields are also deduced. In addition, the methodology is fitted to the context of measurement campaigns which impose simplicity of implementation, auto-calibration, and portability.

Camera internal geometry models are achieved a priori in the laboratory and validated to ensure a certain accuracy in the peripheral parts of the all-sky image. Then, stereophotogrammetry with dense 3-D reconstruction is applied with cameras spaced $150 \mathrm{~m}$ apart for two validation cases. The first validation case is carried out with cumulus clouds having a cloud base height at $1500 \mathrm{~m}$ a.g.l. The second validation case is carried out with two cloud layers: a cumulus fractus layer with a base height at $1000 \mathrm{~m}$ a.g.l. and an altocumulus stratiformis layer with a base height of $2300 \mathrm{~m}$ a.g.l. Velocity fields at cloud base are computed by tracking image rectangular patterns through successive shots. The height uncertainty is estimated by comparison with a Vaisala CL31 ceilometer located on the site. The uncertainty on the horizontal coordinates and on the velocity field are theoretically quantified by using the experimental uncertainties of the cloud base height and camera orientation. In the first cumulus case, segmentation of the image is performed to iden-
\end{abstract}

tify individuals clouds in the cloud field and determine the horizontal positions of the cloud centers.

\section{Introduction}

Understanding cloud physical mechanisms is essential for understanding climate and meteorological processes. On climate scales, it is recognized that clouds are a major source of incertitude in atmospheric models (IPCC, 2013), whether for the energy balance or water cycle. Yet, many aspects of cloud's life cycle are still not understood by the scientific community (Stevens and Feingold, 2009), hence the need for measurement tools allowing cloud monitoring, particularly in a Lagrangian sense.

At present, the instruments most frequently used for remote sensing of clouds from the ground are ceilometers, lidars and cloud radars. Ceilometers and lidars estimate the base height and thickness of several cloud layers. Cloud radars have the same capacities, but are also able to obtain information on the nature of the condensed elements in the cloud (crystals, droplets), as well as their vertical velocities. These ground-based remote sensing instruments are generally oriented towards the zenith and have a narrow field of view. Cloud radars rotate to reconstruct the cloud field; however, the minimum period to complete $360^{\circ}$ sweep is a limiting factor for following a cloud field in real time (Borque et al., 2014). Stereophotogrammetry based on a network of all-sky cameras yields three-dimensional information by matching points across stereo images and using triangulation. These techniques provide an inexpensive method to study the three-dimensional organization of a cloud field. 
The use of all-sky cameras makes it possible to widen the field of view.

Stereophotogrammetry applications for use in meteorology have existed since the beginning of analogue photography (Koppe, 1896; Bradbury and Fujita, 1968), and more recently digital cameras have been used (Allmen and Kegelmeyer, 1997). In the recent years, several technological advances have been made in camera lenses, image resolution, network communications, computational power and cost reduction. Moreover, major computational improvements have been made in computer vision algorithms, especially in multi-vision reconstruction methods (e.g., OpenCV library Bradski and Kaehler, 2008). It is now possible to achieve cloud automatic 3-D reconstruction by stereophotogrammetry relatively cheaply.

Recent studies on this topic generally use conventional or wide-angle lenses to calculate macroscopic characteristics of a cloud field, such as cloud base heights and cloud layer horizontal velocities. Seiz (2003) uses a pair of wideangle cameras spaced $800 \mathrm{~m}$ apart and pointing to the zenith to calculate the height of the cloud base. The orientation of the cameras is done using the stars. The errors obtained are about $5 \%$ for mid-altitude clouds at $4000 \mathrm{~m}$ a.s.l. Hu et al. (2009) use conventional cameras spaced $1.5 \mathrm{~km}$ apart and oriented to mountains to study the three-dimensional organization of orographic convection. The orientation of the cameras is achieved using elements of the landscape. Öktem et al. (2014) are interested in the height of maritime clouds with cameras spaced about $900 \mathrm{~m}$ apart. The cameras are oriented towards the horizon. They obtained an error in cloud base height of $2 \%$ for low-layer clouds and $8 \%$ for cirrocumulus by comparison with lidar measurements. They also calculate a horizontal velocity field that they compare to the data from a radiosonde. In their case, the orientation of the cameras is achieved by using the position of the sun and the horizon line. In all these previous publications, triangulation is based on the matching of corresponding pixels through the stereo images by manual or automatic methods. In Janeiro et al. (2014), the cloud ceiling information for VFRs (visual flight rules) is calculated by matching a zenith-centered sub-part of the initial stereo images. The authors use low-cost consumer cameras that are oriented towards zenith and spaced about $30 \mathrm{~m}$ apart. The orientation of the cameras is achieved using the stars. For clouds under $1500 \mathrm{~m}$ a.g.l., which are of prime interest for VFR applications, results at zenith point show good agreement with lidar measurements in single cloud layer situations.

The first study using all-sky cameras in stereophotogrammetry for meteorological purposes is performed by Allmen and Kegelmeyer (1997) to calculate the cloud base height, but temporal synchronization constraints did not allow for usable information to be obtained. More recently, in order to forecast intra-hour solar irradiance, Nguyen and Kleissl (2014) use their own high-resolution all-sky cameras, providing very precise equisolid projection. The cameras are spaced
$1230 \mathrm{~m}$ apart and the authors use the position of the solar disk to determine orientation. Clouds are filtered in the images with saturation value and cloud base height is determined by plane-sweeping across the stereo images. The results are compared to ceilometer with $8 \mathrm{~h}$ time series. Residual mean square deviation of $7 \%$ for cloud base height at $5000 \mathrm{~m}$ a.s.l. is obtained. Three-dimensional reconstruction is also performed and height distribution of triangulated pixels is compared to ceilometer time series showing good agreement. Recently, Beekmans et al. (2016) performed a dense 3-D reconstruction from a pair of fisheye lens HD cameras spaced $300 \mathrm{~m}$ apart. The relative orientation of the cameras is estimated using the positions of the stars. This estimation is then refined by an algorithm which automatically matches corresponding stereo pixels. The method is validated by comparison with the data of a ceilometer, a lidar and a cloud radar for a cloud layer of altocumulus stratiformis at about $3000 \mathrm{~m}$. The results show cloud base height relative errors less than $5 \%$. The method is then applied to enable a 3-D reconstruction of a developing cumulus mediocris.

In this paper, we use all-sky stereophotogrammetry to perform geolocation of individual elements of a cloud field in order to follow individual clouds in a Lagrangian way, estimate their morphological characteristics and their evolution in real time. Furthermore, this allows the use of cloud geolocation for cloud airborne measurements. For example, in the case of instrumented unmanned aerial vehicles (UAVs), the GPS coordinates of the target cloud may be communicated in real time to the autopilot. In addition, installation of a camera network for a measurement campaign poses additional challenges. Indeed, it may be difficult, time-consuming, or sometimes impossible to use landscape elements, or the position of the stars. Therefore the methodology, developed in Sect. 2, is based on the principles of simplicity of implementation, auto-calibration, and portability.

Stereophotogrammetry is based on triangulation: knowing the distance between two cameras, their orientation and the angles of incidence of the optical rays emitted by a physical point, it is possible to find the 3-D coordinates of the physical point in a given frame. Thus, several indispensable steps are needed. The calibration of each camera encompasses a mathematical description of the projection of an incident optical ray onto the image. This step is carried out in a laboratory using a test pattern. In our case, we use a generic method to perform all-sky camera calibration developed by Scaramuzza et al. (2006). The calibration of the stereo system consists in knowing the distance between the cameras and the relative orientation of each camera. This step is performed once the cameras are installed on the experimental field. In our methodology, positioning and orientation are achieved with GPS, leveling instruments and visual sight, with no obstacles between the two cameras. Precise relative orientation between the cameras is determined by matching feature points across the stereo images automatically. This is achieved with the SIFT algorithm (Lowe, 2004). The 3- 
D reconstruction step consists in finding for each pixel of the left stereo image, its correspondent in the right stereo image. Three-dimensional information is then calculated by triangulation, involving previously calculated camera internal geometry and orientation. In this work, a dense 3-D reconstruction is performed by using a block-matching method (Szeliski, 2010) on rectified stereo images (undistortion and misalignment corrections). Additionally, the velocity field is estimated by tracking subparts of the initial image through two successive images and combine this information with the cloud height map. In the case where clouds are sufficiently separated to be considered as identifiable objects, we implement image segmentation for individual cloud georeferencing. We use a color filter to extract the cloud contours of the image and use a segmentation algorithm inspired by Suzuki and Abe (1985) to identify cloud objects. Most of the methodology relies on algorithms implemented in open-source software libraries: OcamCalib (Scaramuzza et al., 2006) for camera calibration, and OpenCV (Bradski and Kaehler, 2008) for the other steps. The accuracy depends on the quality of the cameras and the algorithms used, as well as on the distance between the cameras, with the following paradox: the greater the distance between the cameras, the better the accuracy but the more difficult pixel matching is.

In Sect. 3, we present the results comparing cloud base heights to traditional methods as well as georeferencing individual cloud elements and calculating the velocity field. The method is applied with cameras spaced $150 \mathrm{~m}$ apart, for two validation cases. The first validation case is carried out in the context of a moderately convective situation with isolated cumulus clouds with a cloud base height at $1500 \mathrm{~m}$ a.g.l. The second validation case is carried out in a situation where two cloud layers overlap: a layer of altocumulus stratiformis with a base height of $2300 \mathrm{~m}$ a.g.l. and a layer of cumulus fractus with a base height at $1000 \mathrm{~m}$ a.g.l. The height uncertainty is estimated by comparison with a Vaisala CL31 ceilometer located on the site. The uncertainty on the horizontal coordinates is theoretically quantified by using the experimental uncertainties on the height and uncertainties on the orientation of the cameras. In the cumulus case, a segmentation of the image as well as an estimation of the horizontal positions of the cloud centers is carried out. The results are then discussed in Sect. 4.

\section{Material and methods}

\subsection{Material}

In this work, we use two VIVOTEK FE8391-V network fisheye cameras designed for outdoor video surveillance applications (see Fig. 1). The focal length is $1.5 \mathrm{~mm}$ and the field of view is $180^{\circ}$. The digital sensor is a 12-megapixel CMOS, providing in its full resolution a $2944 \mathrm{px} \times 2944 \mathrm{px}$ image. The images are transmitted to a computer by a WiFi local (a)
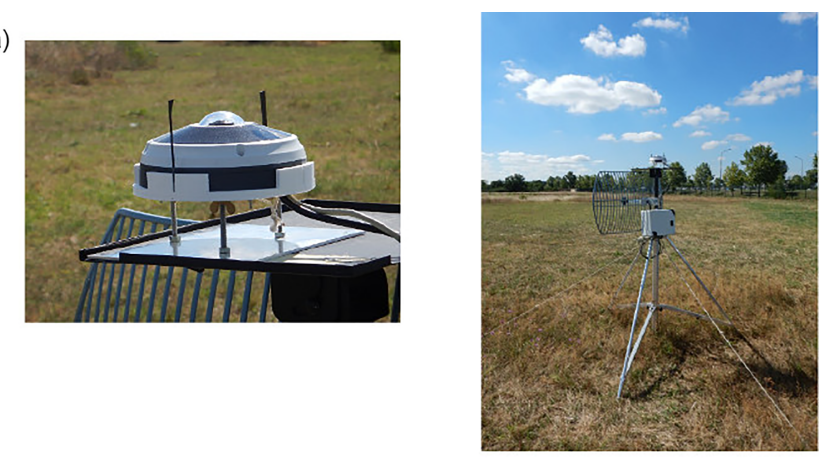

(b)

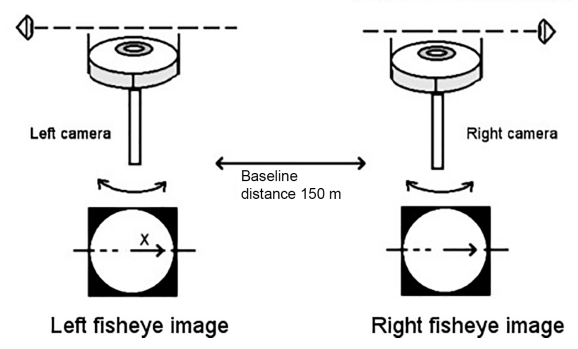

Figure 1. (a) VIVOTEK FE8391-V fisheye camera and installation structure. (b) Vertical sights on the camera housing allow visual inter-camera alignment in the horizontal plane.

network using two directional antennas (TP-Link $2.4 \mathrm{GHz}$ $24 \mathrm{dBi}$ ) with several hundred meters of range. Horizontal leveling is achieved by the use of a bubble level (accuracy ca. $1^{\circ}$ ). The respective positions of both cameras in the Earth frame are evaluated by using GPS, and inter-camera alignment is achieved with vertical sights on the camera housing.

\subsection{Camera projection model, calibration and image undistortion}

In the camera frame, the projection of an optical ray towards a pixel of the image is generally described by a model which depends on intrinsic camera parameters. The camera optical system approaches more or less precisely different types of projections among which the most commonly encountered are the stereographic, equidistant, equisolid, and orthographic projections. In the case of non-scientific cameras, these simple theoretical models are far from sufficient. It is then necessary to use models allowing a better description of the projection by taking imperfections into account (e.g., distortions, offset between optical axis and center of the image, digitization effects). In this article, we use the model proposed by Scaramuzza et al. (2006) to calibrate the cameras. This model was introduced to generically simulate omnidirectional cameras with the property of the single point of view (property generally well approached by a fisheye lens). The intrinsic parameters associated with this model are determined by a calibration step. This calibration is carried out by taking several shots of a flat 2-D chessboard pattern. This flexible technique inspired by Zhang (2000) is adapted in the toolbox OcamCalib for the Scaramuzza model. One of the 

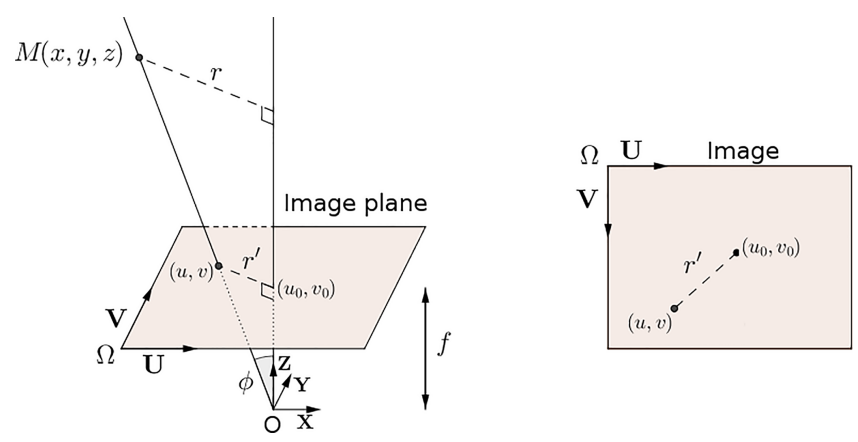

Figure 2. Pinhole projection. Physical point $M$ is projected on $(u, v)$ on the image plane $(\Omega, \boldsymbol{U}, \boldsymbol{V})$. Camera coordinate system is defined by axis $\boldsymbol{X}$ and $\boldsymbol{Y}$, which are colinear to $\boldsymbol{U}$ and $\boldsymbol{V}$, and by axis $\boldsymbol{Z}$, which is the optical axis. Principal point $\left(u_{0}, v_{0}\right)$ is the projection of the optical center $O$ on the image. The radial projected distance on the image is denoted $r^{\prime}$.

advantages of this calibration method is its ease of implementation and its accuracy (e.g., Puig et al., 2012, for a comparative benchmark between several calibration methods for omnidirectional cameras).

\subsubsection{Pinhole camera model}

The starting point is to consider the simplest camera, that is, the pinhole camera. It is a box that allows the light rays to pass through a small hole pierced on one side. On the opposite side of the hole, the inverted scene is projected onto a plate. In order to simplify the way in which this projection is represented, a central symmetry is applied to have a situation in which the image plane and the scene are of the same side with respect to the optical center (Fig. 2). The rectangular image plane has an orthonormal coordinate system $(\Omega, \boldsymbol{U}, \boldsymbol{V})$, where $\boldsymbol{U}$ is the horizontal axis of the image and $V$ the vertical axis of the image. The origin $\Omega$ is located at the upper left corner of the image. The camera reference frame is defined by the orthonormal frame $(O, \boldsymbol{X}, \boldsymbol{Y}, \boldsymbol{Z})$, where $\boldsymbol{Z}$ corresponds to the optical axis directed to the observed scene and $\boldsymbol{X}$ and $\boldsymbol{Y}$ correspond to the $\boldsymbol{U}$ and $\boldsymbol{V}$ axes of the image. The point of intersection of the optical axis with the image is called principal point. It does not necessarily coincide with the center of the image, which is especially the case for nonscientific cameras. In this configuration, if $\left(u^{\prime}, v^{\prime}\right)$ denotes the centered coordinates of a pixel with respect to the principal point, the projection of a physical point $M(x, y, z)$ is given by the following equation:

$$
\left(u^{\prime}, v^{\prime}\right)=(f \tan (\phi) x / r, f \tan (\phi) y / r),
$$

where $r=\sqrt{x^{2}+y^{2}}$ denotes the distance from the physical point to the optical axis and $\phi=\arctan (r / z)$ denotes the angle of incidence of the optical ray. The parameter $f$ is the pinhole camera focal length (expressed in pixels in the case of a digital camera). Thus, if $(u, v)$ denotes the pixel associ-

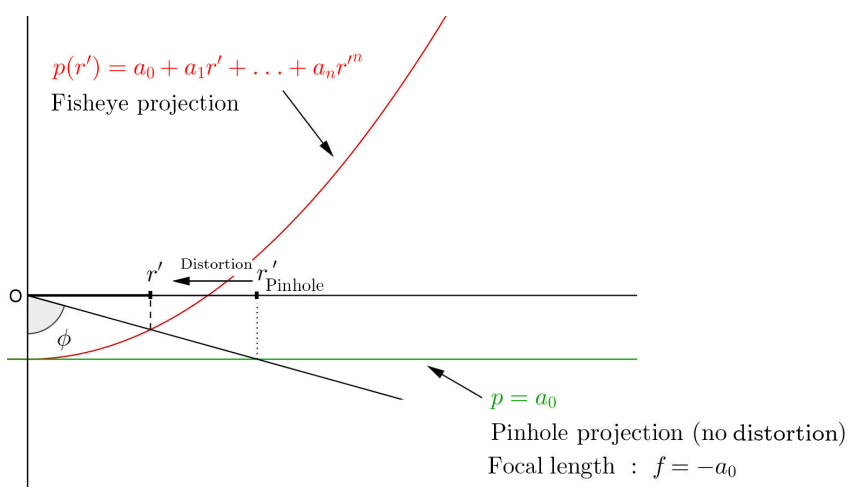

Figure 3. Radial distortion modelization in Scaramuzza et al. (2006) for omnidirectional cameras. Incident angle $\phi$ and projected radial distance $r^{\prime}$ are related by $\tan \phi=-r^{\prime} / p\left(r^{\prime}\right)$. The polynomial function $p$ is represented by the red curve. The case where there is no distortion (i.e., pinhole projection $r^{\prime}=f \tan \phi$ ) corresponds to a constant polynomial function $p=a_{0}$ represented by the green line.

ated with the $M(x, y, z)$ point in the frame of the image, the projection is defined by

$(u, v, 1)^{\mathrm{T}}=\left(\begin{array}{ccc}1 & 0 & u_{0} \\ 0 & 1 & v_{0} \\ 0 & 0 & 1\end{array}\right)\left(u^{\prime}, v^{\prime}, 1\right)^{\mathrm{T}}$,

where $\left(u_{0}, v_{0}\right)$ contains the coordinates of the principal point. We denote $G_{\text {perspective }}^{f, u_{0}, v_{0}}$ the projection function of parameters $\left\{f, u_{0}, v_{0}\right\}$ which maps a physical point $M(x, y, z)$ to a pixel $(u, v)$. The reciprocal projection is denoted by $G_{\text {perspective }}^{-1 f, u_{0}}$. It maps an optical ray $\{\lambda(x, y, 1), \lambda \in \mathbb{R}\}$ to a pixel $(u, v)$.

\subsubsection{Omnidirectional Scaramuzza model}

Under the axisymmetric assumption, and if $r^{\prime}$ denotes the distance between $(u, v)$ and the principal point, Eq. (1) can be generalized to

$$
\left(u^{\prime}, v^{\prime}\right)=\left(r^{\prime}(\phi) x / r, r^{\prime}(\phi) y / r\right) .
$$

The distance $r^{\prime}$ in pixels depends on $\phi$ and characterizes the radial distortions. These distortions are preponderant in a fisheye lens. This is the reason why the function $r^{\prime}(\phi)$ is called representation function of the fisheye lens. In the Scaramuzza model, this function is implicitly defined by the relation $\tan \phi=-r^{\prime} / p\left(r^{\prime}\right)$, where $p\left(r^{\prime}\right)$ is a polynomial function $p\left(r^{\prime}\right)=a_{0}+a_{1} r^{\prime}+\ldots+a_{n} r^{\prime \prime}$ (Fig. 3). The tangential distortions are taken into account linearly by an additional correction step (parameters $c, d$ and $e$ ). Thus, if $(u, v)$ denotes the pixel associated with the $(x, y, z)$ point in the frame of the image, the projection is defined by

$$
(u, v, 1)^{\mathrm{T}}=\mathbf{M}\left(u^{\prime}, v^{\prime}, 1\right)^{\mathrm{T}}=\left(\begin{array}{ccc}
1 & e & u_{0} \\
d & c & v_{0} \\
0 & 0 & 1
\end{array}\right)\left(u^{\prime}, v^{\prime}, 1\right)^{\mathrm{T}} .
$$



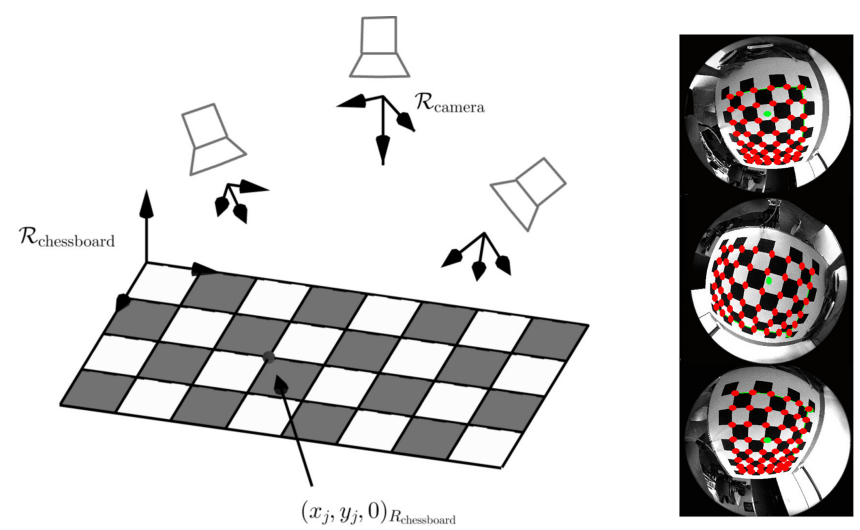

Figure 4. Calibration procedure by multiple views of the same chessboard. The procedure is automatized by using an algorithmic corner detection. The camera projection function is estimated with the OcamCalib toolbox following Scaramuzza et al. (2006) modelization.

We denote $G_{\text {fisheye }}^{\mathbf{M}, a_{0}, \ldots, a_{n}}$ the fisheye projection function of intrinsic parameters $\left\{\mathbf{M}, a_{0}, \ldots, a_{n}\right\}$.

\subsubsection{Camera calibration method}

The camera calibration determines the camera intrinsic parameters $\left\{\mathbf{M}, a_{0}, \ldots, a_{n}\right\}$. To do this, we use $N$ shots of a chessboard with $K_{1} \times K_{2}$ corners (intersections between black and white tiles - Fig. 4 ). We denote by $\mathcal{R}_{\text {chessboard }}$ a coordinate system such that the origin is located on one of these corners, and that the horizontal axes coincide with the chessboard lines. For each shot $i$, and for each chessboard corner $\left(x_{j}, y_{j}, 0\right)_{\mathcal{R}_{\text {chessboard }}}^{\mathrm{T}}$, we have the relation

$\left(u_{i j}, v_{i j}\right)^{\mathrm{T}}=G_{\text {fisheye }}^{\mathbf{M}, a_{0}, \ldots, a_{n}}\left(\mathbf{R}_{i}\left(x_{j}, y_{j}, 0\right)_{\mathcal{R}_{\text {chessboard }}}^{\mathrm{T}}+\boldsymbol{T}_{i}\right)$

$i=1, \ldots, N \quad j=1, \ldots, K_{1} \times K_{2}$,

where $\left(u_{i j}, v_{i j}\right)$ denotes corners positions on the image, $\mathbf{R}_{i}$ the rotation from the camera frame to $\mathcal{R}_{\text {chessboard }}$ and $\boldsymbol{T}_{i}$ the translation between the optical center of the camera and the origin of $\mathcal{R}_{\text {chessboard }}$. The calibration is based on the following steps using the toolbox OcamCalib:

1. For each shot $i$, corners are automatically detected in the image using the intensity gradient specific signal and the pattern of the board (Fig. 4 bottom). This process gives $\left(u_{i j}, v_{i j}\right)$ values.

2. In the nonlinear system (Eq. 5), the values of $\left(u_{i j}, v_{i j}\right)$ and $\left(x_{j}, y_{j}\right)$ are known and the system is overdetermined for sufficiently large values of $N$ and $K_{1} \times$ $K_{2}$. Parameters $\left\{\mathbf{M}, a_{0}, \ldots, a_{n},\left\{\mathbf{R}_{i}, \boldsymbol{T}_{i}, \forall i=1 \ldots N\right\}\right\}$ are determined by using a Marquardt-Levenberg method.

\subsubsection{Undistortion}

In order to produce undistorted images, the scene is reprojected according to a conventional centered perspective projection of focal length $f$. During this reprojection, we move from a circular fisheye image to a square image of size $N_{\mathrm{px}} \times N_{\mathrm{px}}$. The intensity of each pixel of the undistorted image is calculated according to the relationship

$$
\begin{gathered}
\operatorname{RGB}_{\text {undistorted }}\left(u_{\text {undistorted }}, v_{\text {undistorted }}\right)= \\
\operatorname{RGB}_{\text {fisheye }}\left(G_{\text {fisheye }}^{\mathbf{M}, a_{0}, \ldots, a_{n}} \cdot G_{\text {perspective }}^{-1 f, N_{\mathrm{px}} / 2, N_{\mathrm{px}} / 2}\right. \\
\left.\left(u_{\text {undistorted }}, v_{\text {undistorted }}\right)\right) .
\end{gathered}
$$

In this transformation, the peripheral areas are mapped from a given region of the fisheye image onto a larger projection area in the rectified image, producing the blur effect. Note that the values of $f$ and $N_{\mathrm{px}}$ can be freely chosen. The field of view of undistorted images $\mathrm{FOV}_{\text {undistorted }}=2 \arctan \frac{N_{\mathrm{px}}}{2 f}$ will depend on these values. The smaller the value of $f$, the larger the field of view, but the more interpolated areas occupy an important part of the image.

\subsection{Orientation, stereo calibration and rectification}

At the end of the previous step, we are able to produce two undistorted stereo images. They are square images of the same size $N_{\mathrm{px}} \times N_{\mathrm{px}}$, for which the center of the image and the principal point coincide, and which would have been taken by two pinhole cameras with the same focal length $f$. The next step consists in orienting them with respect to each other as accurately as possible. To achieve this, Hu et al. (2009) use landscape features and Öktem et al. (2014) use the horizon line. Seiz (2003) and Beekmans et al. (2016) use the positions of the stars that allows for determining the orientation of each camera. In addition, they add an algorithmic correction step based on SIFT stereo-pixel-matching algorithm (Lowe, 2004). In our work, we develop a visual orientation method assuming that there is no visual obstacle between the two cameras. Like Beekmans et al. (2016), this initial orientation is refined by an algorithmic step.

\subsubsection{Orientation and stereo calibration}

The cameras optical axis are oriented towards the zenith. The image planes are at the same altitude, and the horizontal axes of the undistorted images are aligned. This theoretical orientation of the all-sky stereo system is called frontally aligned (Fig. 5). From the GPS coordinates, the distance $b=O_{1} O_{2}$ between the cameras and the angle of deviation from the North $\beta={\widehat{N O_{1} O_{2}}}_{2}$ are calculated with Haversine formulas. Initial orientation of the cameras is previously described in Fig. 1 and gives an orientation of the cameras close to the ideal frontally aligned orientation. However, this procedure is not sufficient to perform an accurate 3-D reconstruction 


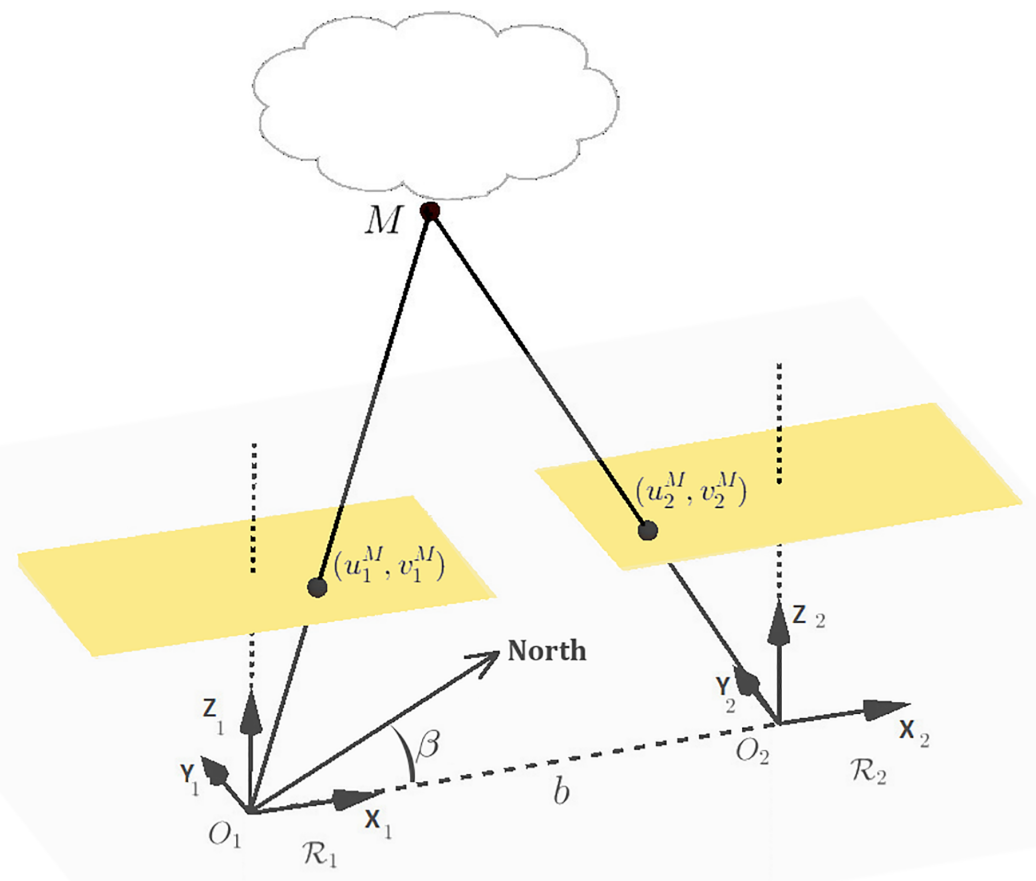

Figure 5. Ideal camera configuration. Camera coordinate systems are frontally aligned with optical axis $z_{1,2}$ oriented towards zenith. Optical centers $O_{1,2}$ are in the same altitude plane. The baseline distance is denoted $b$ and North bearing of $O_{1} O_{2}$ axis is denoted $\beta$. In this ideal configuration, assuming that we have identical pinhole centered cameras, corresponding pixels $\left(u_{1}^{M}, v_{1}^{M}\right)$ and $\left(u_{2}^{M}, v_{2}^{M}\right)$ are row-aligned on the imagers $\left(\right.$ i.e., $\left.v_{1}^{M}=v_{2}^{M}\right)$.

which needs row alignment of corresponding stereo pixels in the stereo images (see Fig. 5). A refining algorithmic step to calculate the precise relative orientation of the cameras and consequently rectify the stereo images is then required. This procedure is usually referred to as stereo calibration and consists of calculating the components of the relative rotation $\mathbf{R}$ and the relative translation $\boldsymbol{T}=O_{1} O_{2}$ between camera frames such as $(x, y, z)_{\mathcal{R}_{1}}^{\mathrm{T}}=\mathbf{R}(x, y, z)_{\mathcal{R}_{2}}^{\mathrm{T}}+\boldsymbol{T}$.

Stereo calibration is based on the concepts and theorems of epipolar geometry. In particular, in the case of pinhole cameras with the same focal length $f$, it exists a constant matrix $3 \times 3$ of rank 2 denoted $\mathbf{E}$ and called essential matrix. This matrix only depends on $\mathbf{R}$ and $\boldsymbol{T}$ and verifies the following constraint:

$\left(u_{2}^{\prime M}, v_{2}^{\prime M}, 1\right) \mathbf{E}\left(u_{1}^{\prime M}, v_{1}^{\prime M}, 1\right)^{\mathrm{T}}=0$

for all pixels $\left(u_{1}^{M}, v_{1}^{M}\right)$ from the left stereo image, and $\left(u_{2}^{M}, v_{2}^{M}\right)$ from the right stereo image representing the same physical point $M$. We use the following stereo calibration methodology:

1. From the undistorted stereo images, retrieve a set of stereo matching pixels with the SIFT algorithm (Lowe, 2004).
2. Using the pairings of step 1, solve the overdetermined system (Eq. 7) whose unknowns are the coefficients of the matrix $\mathbf{E}$. We use a least median of squares (LMEDS) regression, which avoids being affected by outliers. The matrix $\mathbf{E}$ is determined to within a scalar factor.

3. Calculate $\mathbf{R}$ and $\boldsymbol{T}$. For this purpose, the following equations are used:

$$
\begin{aligned}
& {\left[\boldsymbol{T}_{\times}\right]^{2}=-\mathbf{E E}^{\mathrm{T}}} \\
& \text { with }\left[\boldsymbol{T}_{\times}\right]=\left(\begin{array}{ccc}
0 & -T_{z} & T_{y} \\
T_{z} & 0 & -T_{x} \\
-T_{y} & T_{x} & 0
\end{array}\right),
\end{aligned}
$$

which give two opposite solutions $\boldsymbol{T}_{+}$and $\boldsymbol{T}_{-}$and

$\mathbf{R}=\frac{1}{\|\boldsymbol{T}\|^{2}}\left(\left[\begin{array}{lll}\boldsymbol{E}_{2} \times \boldsymbol{E}_{3} & \boldsymbol{E}_{3} \times \boldsymbol{E}_{1} & \boldsymbol{E}_{1} \times \boldsymbol{E}_{2}\end{array}\right] \pm\left[\boldsymbol{T}_{\times}\right] \mathbf{E}\right)$,

where $\boldsymbol{E}_{k}$ denotes the $k$ th column of the $\mathbf{E}$ matrix. The uniqueness of the solution is obtained by requiring the scene to be located in front of the cameras as well as the constraint $\|\boldsymbol{T}\|=b$. 
4. Corrective rotations $\mathbf{R}_{1}$ and $\mathbf{R}_{2}$ are defined by using $\mathbf{R}$ and $\boldsymbol{T}$ such that

$$
\mathbf{R}_{1}=\mathbf{R}_{\text {rect }}^{-1} \text { and } \mathbf{R}_{2}=\mathbf{R}_{\text {rect }}^{-1} \mathbf{R}
$$

where $\mathbf{R}_{\text {rect }}=\left(\boldsymbol{e}_{1}, \boldsymbol{e}_{2}, \boldsymbol{e}_{3}\right)$ is a rotation matrix such as $\boldsymbol{e}_{1}$ is oriented in the same direction of $\boldsymbol{T}$, and $\boldsymbol{e}_{2}$ is orthogonal to $\boldsymbol{e}_{1}$ and to the left camera optical axis.

5. Consistency step: initial visual orientation of the cameras is achieved to be as close as possible to the frontally aligned relative orientation (i.e., $\mathbf{R} \simeq \mathrm{Id}$ and $\boldsymbol{T} \simeq(b, 0,0)^{\mathrm{T}}$; see Sect. 2.3). In our algorithm, several estimations of the essential matrix $\mathbf{E}$, and consequently $\mathbf{R}$ and $\boldsymbol{T}$, are achieved to avoid incorrect solutions which are due to erroneous or imprecise matches in the SIFT procedure. These estimations of $\mathbf{E}$ are obtained by using several subsets of the matching pixel set given by the SIFT procedure. Estimations of $\mathbf{E}$ matrix, which are not coherent with the $\mathbf{R} \simeq \mathrm{Id}$ and $\boldsymbol{T} \simeq(b, 0,0)^{\mathrm{T}}$ hypothesis, are then rejected. Among the coherent estimations, we choose the one that leads to minimal corrective rotations.

\subsubsection{Rectification}

We use $\mathbf{R}_{1}$ and $\mathbf{R}_{2}$ to produce undistorted rectified images; that is, the images that would have been produced by perfectly aligned pinhole cameras. These images are produced from all-sky original images by the following transformation:

$$
\begin{gathered}
\operatorname{RGB}_{\text {rectified }}^{\mathrm{CAM1} 2}\left(u_{\text {rectified }}, v_{\text {rectified }}\right)= \\
\operatorname{RGB}_{\text {fisheye }}^{\text {CAM1,2 }}\left(G_{\text {fisheye }}^{\text {intrinsicparamsCAM1,2 }} \cdot G_{\text {perspective }}^{-1 f, N_{\mathrm{px}} / 2, N_{\mathrm{px}} / 2}\right. \\
\left.\cdot \mathbf{R}_{1,2}\left(u_{\text {rectified }}, v_{\text {rectified }}, 1\right)\right) .
\end{gathered}
$$

\subsection{Three-dimensional reconstruction}

Three-dimensional reconstruction is obtained by triangulation from two pixels $\left(u_{1}^{M}, v_{1}^{M}\right)$ and $\left(u_{2}^{M}, v_{2}^{M}\right)$ which are known to represent the same physical point $M$. Indeed, knowing the projection functions of each camera, their relative orientations, and the distance between the cameras, it is possible to estimate the point of intersection of the optical rays in a given reference frame. Working directly with the rectified images make this calculation easier because we have a simple theoretical standard situation: identical pinhole images in a frontally aligned orientation (Fig. 5 right). In this case, two matching pixels are located on the same row in the image matrices (i.e., $v_{1}^{M}=v_{2}^{M}$ ). Then, the coordinates $\left(x_{M}, y_{M}, h_{M}\right)$ in the rectified frame of the left camera are given by

$h_{M}=\frac{f b}{u_{2}^{M}-u_{1}^{M}}=\frac{f b}{\delta^{M}}$,

$x_{M}=h_{M} \frac{u_{1}^{\prime M}}{f}, \quad y_{M}=h_{M} \frac{v_{1}^{\prime M}}{f}$,

where $\delta^{M}=u_{2}^{M}-u_{1}^{M}$ is called disparity and is linearly related to $h$ through the baseline distance between the cameras $b$, and the focal distance $f$.

In addition, a dense 3-D reconstruction of the observed scene assumes that one is able to generate a dense matching of corresponding pixels across the stereo images. This is called the dense stereo matching problem. In the case of rectified images, this problem is greatly simplified by the fact that $v_{1}^{M}=v_{2}^{M}$ and thus becomes a one-dimensional problem. In this case, a very common method is the blockmatching algorithm (Szeliski, 2010), which relies on finding maximum correlations between neighborhoods of pixels across the stereo images. This algorithm is implemented in the OpenCV library (Bradski and Kaehler, 2008), and is able to describe finely the variations of altitude. However, it generates noise/speckles in the weakly textured image part, which is a disadvantage for the type of objects that we consider (clouds, blue sky background, sun). To avoid this effect, we use several techniques:

- Adjusting algorithm parameters:

- Disparity range is limited during the pixelmatching process by setting minimum and maximum bounds for cloud height detection. Note that disparity bounds are related to height detection bounds with equation (Eq. 12), even if this relationship becomes less relevant for larger incident angles for which larger horizontal errors occur.

- Window correlation size is adjusted to prevent speckles.

- Smoothing the signal by reducing the size of the image while taking advantage of the subpixel resolution of the algorithm.

- Using blue sky filtering: we process the altitude map by filtering the blue sky areas. We use image conversion in the HSV (hue, saturation, value) color management system. The hue values ranging from 170 to $280^{\circ}$ (from cyan to violet) are filtered.

\subsection{Velocity field}

The estimation of the cloud field horizontal velocity is carried out by using two successive rectified images, $I^{t_{1}}$ and $I^{t_{2}}$, coming from the same camera. Using cross-correlation techniques, the displacement of the cloud field from one image to another is evaluated in pixel units. This displacement 

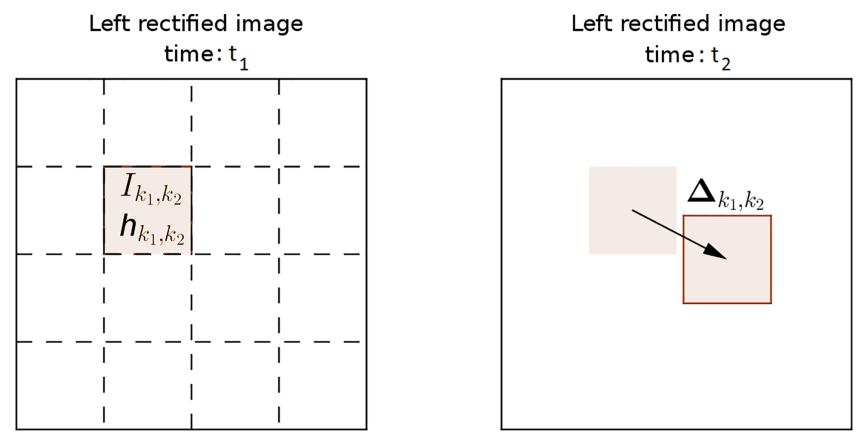

Figure 6. Multiblock tracking algorithm for cloud field velocity estimation. For each block $I_{k_{1}, k_{2}}$, the velocity vector is computed by using the displacement vector $\Delta_{k_{1}, k_{2}}$ expressed in pixels and the median altitude $h_{k_{1}, k_{2}}$. Displacement vector is computed by using the Lewis (1995)'s matching template algorithm. Computations are based on two successive rectified images: in our case we use the left rectified image at times $t_{1}$ and $t_{2}$.

on the image is converted into velocity by using the previously calculated height map. In practice, the initial image is divided into rectangular blocks $I_{k_{1}, k_{2}}^{t_{1}}$ indexed by the subscripts $k_{1,2}$ (Fig. 6). The median of heights $h_{k_{1}, k_{2}}$ is assigned to these blocks based on the cloud height map. The translation in number of pixels of each block through two successive shots is denoted by $\Delta_{k_{1}, k_{2}}$. It is related to the block mean horizontal velocity $\left(v_{k_{1}, k_{2}}^{x}, v_{k_{1}, k_{2}}^{y}\right)$ by

$v_{k_{1}, k_{2}}^{x}=\frac{h_{k_{1}, k_{2}}}{f} \frac{\Delta_{k_{1}, k_{2}}^{u}}{\Delta t}$,

$v_{k_{1}, k_{2}}^{y}=\frac{h_{k_{1}, k_{2}}}{f} \frac{\Delta_{k_{1}, k_{2}}^{v}}{\Delta t}$

where $\Delta t=t_{1}-t_{2}$ is the time between two shots. Calculating $\Delta_{k_{1}, k_{2}}$ is to determine the position of a $I_{k_{1}, k_{2}}^{t_{1}}$ template in the $I^{t_{2}}$ image. This generic computer vision problem is called template matching. A method developed by Lewis (1995) and based on the normal cross correlation index allows performing this search with a low algorithmic cost in simple cases (no rotation, no scaling). This algorithm is available in the OpenCV library (Bradski and Kaehler, 2008).

Note that the technique used here is similar to that used by Janeiro et al. (2014), which evaluates the displacement of a single block centered on the principal point through two images. In our case, the approach is multiblock, which generates dispersion but makes it possible to estimate the velocities of multiple cloud layers.

\subsection{Segmentation and cloud identification}

Segmentation techniques are used in computer vision problems to identify objects in an image. In our case, the main interest of this technique is to identify and georeference individual clouds when the situation allows it. The method that we present here is a contour-based method involving blue sky filtering which supposes that the clouds are separated (e.g., cumulus cloud field) and that they do not overlap on the image due to projection (this would result in merged contours). Segmentation is achieved with the following steps:

1. Production of a binarized image from blue sky filter (Sect. 2.4).

2. Contour detection and segmentation using the binarized image: we use a contour finding algorithm implemented in OpenCV library and inspired by Suzuki and Abe (1985).

3. Filtering non-significant/noisy contours: we eliminate contours with a low inside area, and with a low number of inner triangulated pixels.

4. Filtering sun: we use a threshold on altitude to remove the sun.

Each segmented region contains pixels that have been triangulated in the 3-D reconstruction process. This allows assigning $(x, y, z)$ coordinates for each triangulated pixel. In order to avoid outliers the center of each segmented cloud, and the cloud base height is estimated with

$$
\begin{aligned}
& x_{\text {center }}=\frac{q_{5}(\underline{x})+q_{95}(\underline{x})}{2}, \quad y_{\text {center }}=\frac{q_{5}(\underline{y})+q_{95}(\underline{y})}{2}, \\
& z_{\text {cloudbase }}=q_{10}(\underline{z})
\end{aligned}
$$

where $\underline{x}, \underline{y}, \underline{z}$ corresponds to coordinates of all triangulated pixels within the segmented region. The notation $q_{r}(\underline{x})$ (or $\underline{y}$ and $z$ ) denotes the $r$ th quantile of $x$ values (or $y$ and $z$ ) within the segmented region.

\subsection{Uncertainty estimation}

Theoretically, in a frontally aligned pinhole stereo system, the uncertainty on height $\sigma_{h}$ can be related to the uncertainties on the position of corresponding pixels $\left(u_{1}, v\right),\left(u_{2}, v\right)$, given by the sensitivity equation

$\sigma_{h}=\sigma_{\left|u_{1}-u_{2}\right|} \frac{h^{2}}{f b}=\sigma_{\delta} \frac{h^{2}}{f b}$.

where $\sigma_{|\delta|}=\sigma_{\left|u_{1}-u_{2}\right|}$ represents the uncertainty on disparity (Sect. 2.4). This equation shows that uncertainty decreases linearly as the baseline distance $b$ increases, until a distance where the quality of the stereo-pixel-matching degrades. On the other hand, $\sigma_{h}$ quadratically increases with increasing heights.

In a practical way, the uncertainty related to the 3-D reconstruction of the cloud field in the Earth's frame has several components: camera resolution, intrinsic projection/calibration model, position and orientation of the cameras/stereo calibration, and pixel matching. We quantify the overall uncertainty on cloud base height experimentally. In 
Table 1. OcamCalib calibration results for cameras 1 and 2. Parameters are described in Eqs. (3) and (4) in Sect. 2.2.

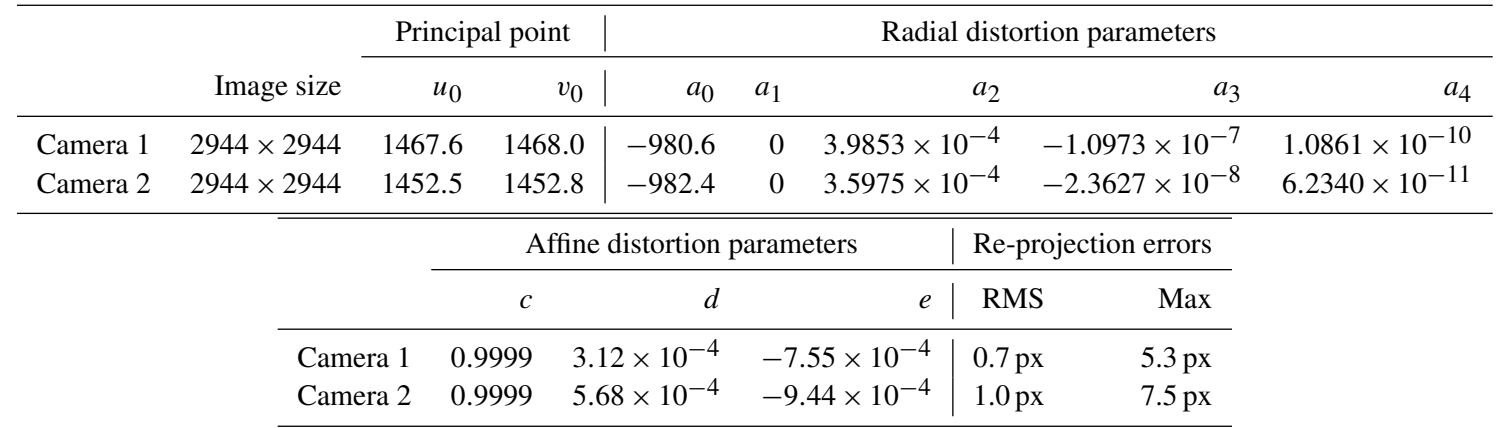

this work, we use a Vaisala CL31 ceilometer, collocated with the all-sky stereo system, as the reference instrument. It provides information by measuring the cloud base height at the zenith and identifies up to three cloud layers. Several aspects must be identified before comparing ceilometer and all-sky stereo system results:

1. There is spatial inter-cloud and intra-cloud variability of the cloud base height.

2. All-sky stereo system computes heights coming from the base as well as the sides of the clouds.

3. Ceilometer provides a point value at zenith, while the cameras provide a spatial map of the heights.

4. All-sky stereo system can recover multiple cloud heights only if it can see them.

Several methodologies can be used to compare all-sky spatial data to ceilometer temporal data. A comparison of height measurements at zenith when the picture is taken allows estimating uncertainty on height $\sigma_{h}$, although this method is limited because it does not represent the uncertainty on the peripheral parts of the image. Another way is to compare the height-frequency histograms obtained by the all-sky stereo system (heights calculated for a scene) with the distribution of the heights obtained by the ceilometer (centered time series). The distribution peaks represent the representative height of the cloud base for a given cloud layer. The thickness associated with these peaks is due to the abovementioned uncertainties and cloud base variability. The error is estimated by comparing the peak positions and the standard deviations of the distributions around these peaks.

In the Earth frame, the uncertainty on $(x, y)$ position can be deducted from uncertainties on height $\sigma_{h}$, polar angle $\sigma_{\phi}$, and azimuthal angle $\sigma_{\theta}$. Indeed, in spherical coordinates we have $x=\rho \cos \theta \sin \phi, y=\rho \sin \theta \sin \phi, h=\rho \cos \phi$. By denoting $r=\sqrt{x^{2}+y^{2}}=h \tan \phi$, the ground projected distance, we obtain $x=h \cos \theta \tan \phi$ and $y=h \sin \theta \tan \phi$, such that

$$
\begin{aligned}
\sigma_{x}^{2}= & (\cos \theta \tan \phi)^{2} \sigma_{h}^{2}+(h \sin \theta \tan \phi)^{2} \sigma_{\theta}^{2} \\
& +\left(h \cos \theta \cos ^{-2} \phi\right)^{2} \sigma_{\phi}^{2}, \\
\sigma_{y}^{2}= & (\sin \theta \tan \phi)^{2} \sigma_{h}^{2}+(h \cos \theta \tan \phi)^{2} \sigma_{\theta}^{2} \\
& +\left(h \sin \theta \cos ^{-2} \phi\right)^{2} \sigma_{\phi}^{2}, \\
\sigma_{r}^{2}= & \tan ^{2} \phi \sigma_{h}^{2}+h^{2} \cos ^{-4} \phi \sigma_{\phi}^{2} .
\end{aligned}
$$

The angle uncertainties are mainly related to the orientation of the cameras: initial orientation (GPS position and visual sighting) and algorithmic correction in the rectification process (Sect. 2.3). In our study, the estimation of $\sigma_{h}$ is calculated experimentally as mentioned above. The corrective rotations provided by the rectification algorithm in different configurations allows estimating $\sigma_{\theta}$ and $\sigma_{\phi}$, providing $\sigma_{\theta}=\sigma_{\phi}=2^{\circ}$.

\section{Results}

\subsection{Camera calibration}

The OcamCalib toolbox allows computing both camera intrinsic parameters following Scaramuzza's model (Sect. 2.2). This calculation is made from multiple images of a chessboard (30 shots in our case). Validation of the calibration is based on two indicators: the first indicator is the mean reprojection error, which is the mean root square of the difference in pixel units between corner positions as estimated through the calibrated model and those initially detected. The second indicator measures the maximum value of this difference. These values (in pixel units) are compared with the size of the images produced by the cameras: 2944 px $\times 2944$ px. Dispersion can also be quantified by comparing the representation functions determined for both cameras. The calibration parameters are listed in Table 1. Figure 7a shows the $r^{\prime}{ }_{\mathrm{CAM} 1}(\phi)$ representation function of camera 1 , as calculated by the calibration method. This function, which characterizes the projection, is compared with typical fisheye parametric models. The difference between the representation functions 

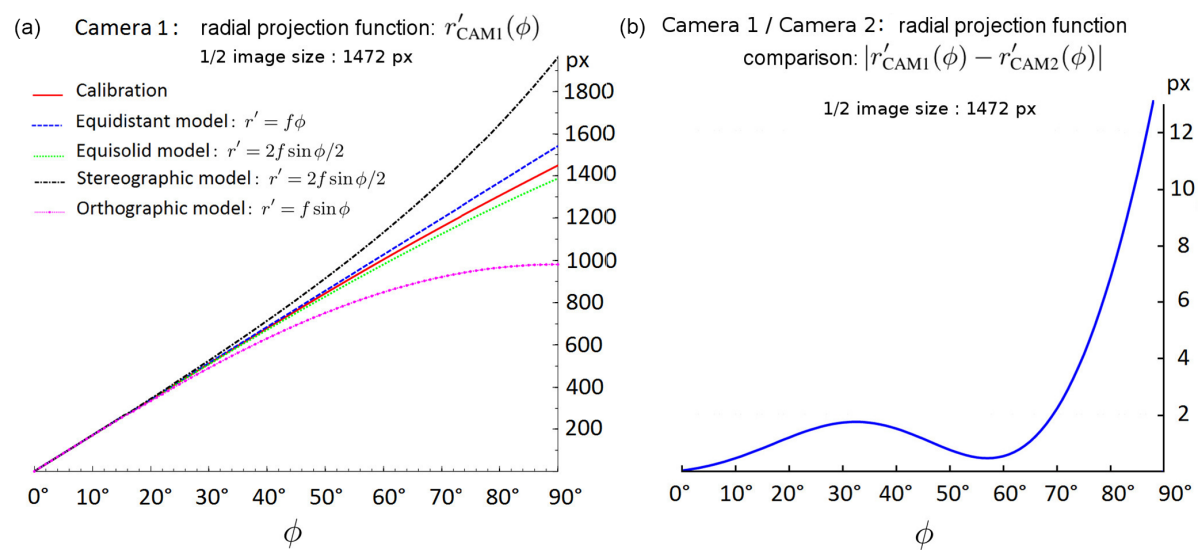

Figure 7. (a) Radial projected distance $r^{\prime}$ as a function of incident angle $\phi$ for VIVOTEK camera 1. Function $r^{\prime}(\phi)$ is called representation function as it characterize the projection. It is compared to the mostly used fisheye parametric representation functions set with $-a_{0}$ value for $f$. (b) difference in pixels between representation functions of cameras 1 and 2 , as a function of incidence angle $\phi$.
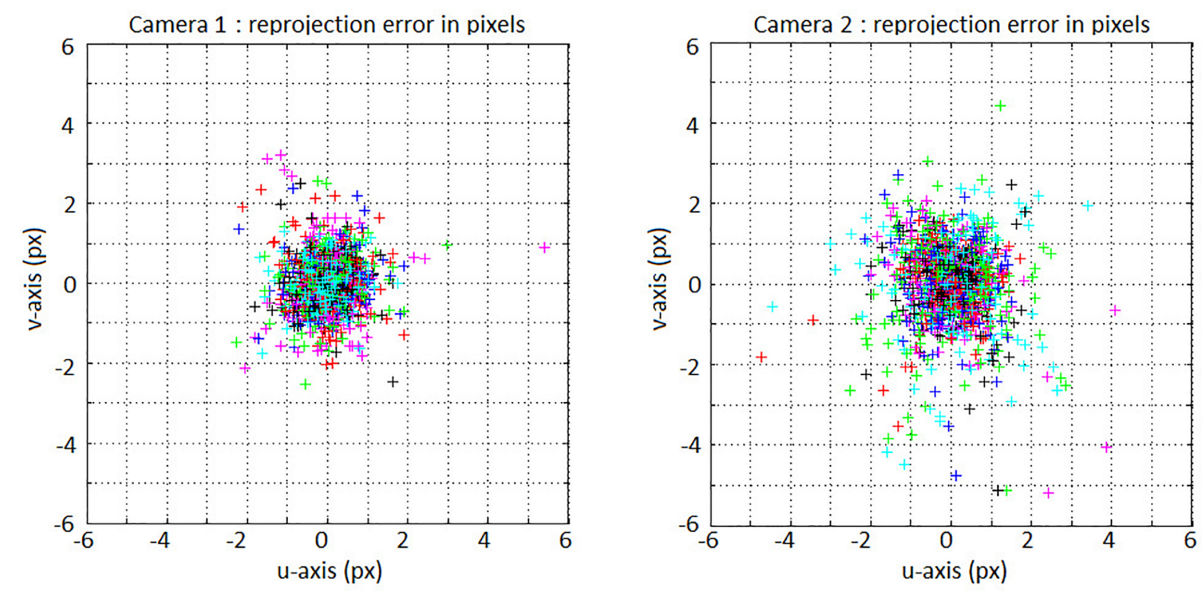

Figure 8. For each view and each chessboard corner (which represents an amount of $30 \times 48$ points), difference between corner position on the image, and corner position computed by re-projection, using OcamCalib calibration results.

of the cameras 1 and 2 is shown in Fig. 7b. The calculation of sensitivity $\mathrm{d} \phi / \mathrm{d} r^{\prime}$ allows estimating the uncertainty on the angle of incidence $\phi$. In our case, this uncertainty varies as a function of $\phi$ between 0.06 and $0.07^{\circ} \mathrm{px}^{-1}$. Finally, Fig. 8 illustrates the dispersion of the reprojection errors for each corner and for each shot.

The result of this calibration shows that both all-sky cameras obtain an intermediate projection between the equidistant and equisolid projections (Fig. 7a), with the difference increasing significantly beyond an incident angle of $50^{\circ}$ $\left(10 \mathrm{px}\right.$ deviation/angular error of $\left.0.65^{\circ}\right)$. This shows that the use of a precise calibration model and method is needed if one wishes to use the peripheral parts of the all-sky image. The difference between the representation functions of the camera 1 and the camera 2 (Fig. 7b) shows that the fisheye projections are almost identical up to an angle of incidence of $70^{\circ}$ ( $2 \mathrm{px}$ deviation), which is an indicator of validity of the calibration. This uncertainty increases significantly beyond angles greater than $80^{\circ}$. The dispersion of the reprojection error is small (Table 1, Fig. 8) with an average reprojection error less than $1 \mathrm{px}$ (i.e., $0.065^{\circ}$ ) and $7 \mathrm{px}$ (i.e., $0.5^{\circ}$ ) maximum deviation.

\subsection{Georeferencing results}

\subsubsection{Validation cases}

We apply the methodology described in Sect. 2 for two types of cloud fields. In both cases, measurements are made on the Météo-France site in Toulouse, France. The baseline distance between the cameras is given by $b \simeq 150 \mathrm{~m}$. The first validation case is carried out in the context of a weakly convective mid-afternoon situation (July 2016). Clouds are cumulus humilis, mediocris and congestus. The cloudiness is around 4 octas. In this case, the cloud base height is around $1500 \mathrm{~m}$ a.g.l., which implies a ratio $h / b \simeq 10$. The second 
Table 2. Description of validation cases.

\begin{tabular}{|c|c|c|}
\hline & Validation case 1 & Validation case 2 \\
\hline Place & \multicolumn{2}{|c|}{ Toulouse - Météo France } \\
\hline Date (UTC) & 8 July 2016 13:55 & 16 June 2016 10:00 \\
\hline Shots & \multicolumn{2}{|c|}{3 shots every $15 \mathrm{~min}$} \\
\hline Baseline distance between cameras, $b$ & \multicolumn{2}{|c|}{$147 \mathrm{~m} \pm 3 \mathrm{~m}$} \\
\hline Type of clouds & $\begin{array}{l}\text { cumulus humilis and } \\
\text { mediocris }\end{array}$ & $\begin{array}{l}\text { cumulus fractus and } \\
\text { altocumulus stratifromis }\end{array}$ \\
\hline Mean cloudiness & $50 \%$ (4 octas) & $75 \%$ (6 octas) \\
\hline Mean cloud base height & $1500 \mathrm{~m}$ a.g.1. & 1000 and $2300 \mathrm{~m}$ a.g.l. \\
\hline Ceilometer & \multicolumn{2}{|c|}{$\begin{array}{c}\text { Frequency: } 1 \mathrm{~min} \text {; start/end time of temporal series: } \\
\quad \pm 15 \mathrm{~min} \text { before and after camera shot }\end{array}$} \\
\hline
\end{tabular}

Table 3. Algorithm parameters.

\begin{tabular}{ll}
\hline Undistortion/rectification algorithms & $\begin{array}{l}\text { Size of undistorted and rectified images: } N_{\mathrm{px}}=2944 \\
\text { Field of view of undistorted/rectified images } 136^{\circ}\end{array}$ \\
\hline Block-matching algorithm parameters & $\begin{array}{l}\text { Size reduction of images: } 512 \times 512 ; \text { max height: } 4000 \mathrm{~m} \text {; min height: } 400 \mathrm{~m} ; \\
\text { correlation window size: } 11 \mathrm{px} \text {; subpixel scale: } 1 / 16\end{array}$ \\
\hline Segmentation/significative contour thresholds & $\frac{\text { inside contour area }\left(\mathrm{px}^{2}\right)}{\text { total image area }}>\frac{1}{1000}, \frac{\text { no. of triangulated pixels in contour }}{\text { no. of pixels in contour }}>75 \%$. \\
\hline
\end{tabular}

validation case occurred around noon, highlighting the detection of multiple cloud layers (June 2016). The clouds are cumulus fractus with cloud base at $1000 \mathrm{~m}$ a.g.l. and altocumulus stratiformis with bases at $2300 \mathrm{~m}$ a.g.l. The cloudiness is about 6 octas. In this case, we have a ratio $h / b \leq 15$. The context of each test case is summarized in Table 2.

\subsubsection{Cloud height map}

For each validation case, we repeat the same procedure three times at intervals of $15 \mathrm{~min}$.

1. Capture and undistortion of the fisheye images (Sect. 2.2).

2. Stereo pixel matching and stereo calibration (Sect. 2.3).

3. Rectification of the undistorted images (Sect. 2.3).

4. Three-dimensional reconstruction and calculation of the height map (Sect. 2.4).

5. Comparison with a \pm 15 min time series from the ceilometer (Sect. 2.7).

Note that in operational situations, the stereo calibration (step 2) does not need to be performed before each shot if the material stays in place. Since we quantify the error associated with the entire methodology, step 2 is re-executed for each shot. In step 4, smoothing and filtering techniques to avoid speckles in non-textured zones are implemented (Sect. 2.4). In particular, $\mathrm{min} / \mathrm{max}$ threshold on heights is set to $h \in[450 \mathrm{~m}, 4000 \mathrm{~m}]$ and a blue-sky filter is implemented. The parameters for image undistortion, rectification, 3-D reconstruction and segmentation are given in Table 3 .

We compare the distributions of the heights obtained with the all-sky stereo system to the ceilometer. The results obtained for the first and second case are presented in Figs. 9 and 10 , respectively, with images spaced $15 \mathrm{~min}$ apart. On those panels, the top row represents the undistorted and rectified images of the left camera at each time interval. The middle row represents cloud height maps. The bottom row represents the distributions of the calculated heights (blue histograms) compared with the ceilometer distributions (red histograms). In addition, ceilometer and all-sky system cloud base height estimations at zenith are given for each time. The comparison of ceilometer and all-sky system height distributions is summarized in Fig. 11.

The results of the cumulus case show that, for cameras spaced $150 \mathrm{~m}$ apart and a cloud base height of about $1500 \mathrm{~m}$ a.g.l., the cloud base height distributions obtained with the all-sky stereo system are similar to the ceilometer. The maximum offset on the distribution peak is about $\pm 150 \mathrm{~m}$, which is $\sigma_{h} / h \simeq 10 \%$ for a $h / b \simeq 10$ ratio. Around these peak values, the data show a standard deviation $\sigma_{h} \simeq$ 

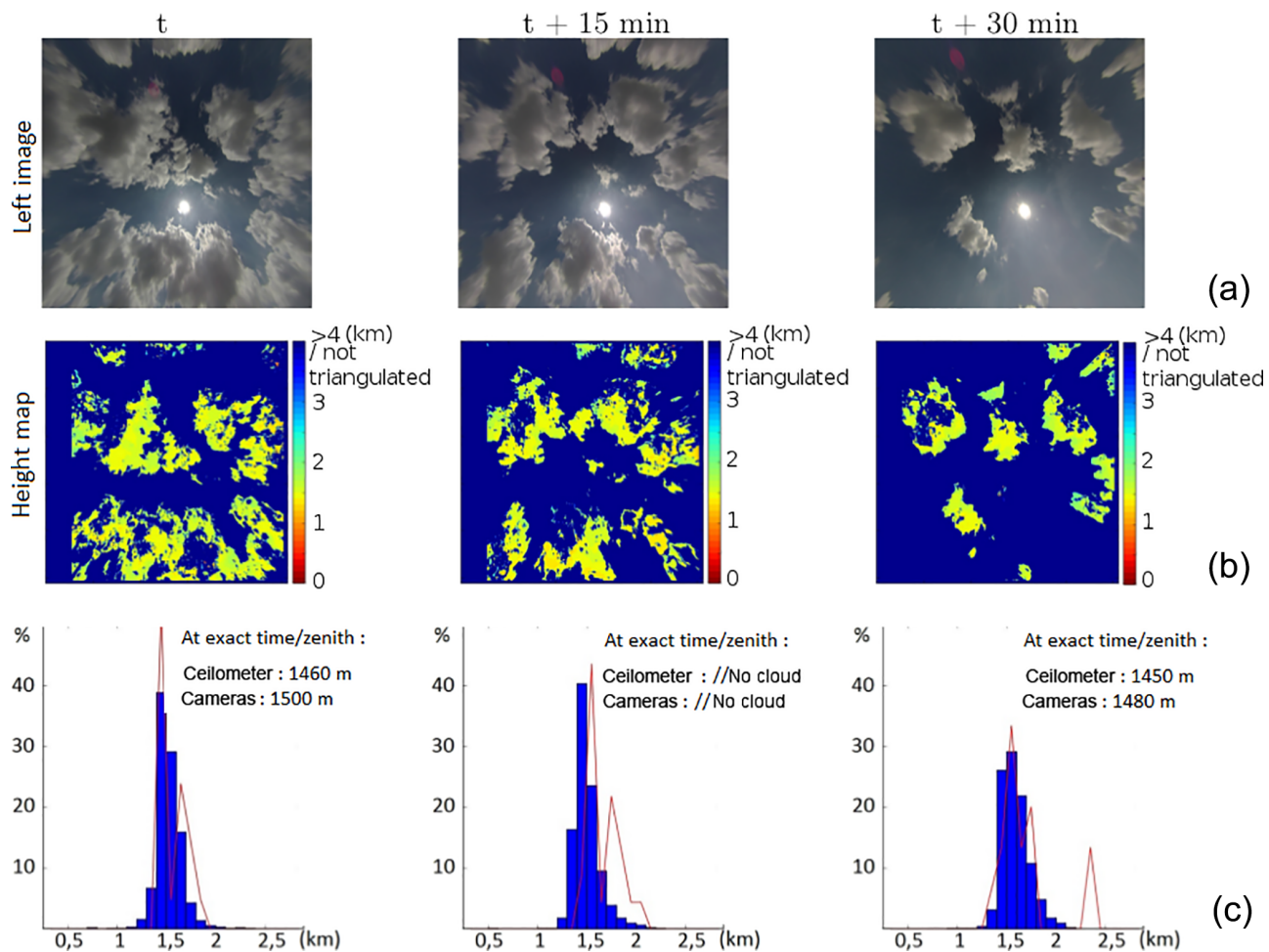

(a)

Figure 9. Cumulus validation case - height map and distribution for three shots evenly spaced of 15 min. Panel (a) represents the left camera images. Panel (b) represents the associated height map computed by the stereovision system. Panel (c) represents the frequency histogram of heights computed by the stereovision system (blue diagram - $100 \mathrm{~m}$ bins). This distribution is compared to the ceilometer frequency histogram (red curve - $100 \mathrm{~m}$ bins).

$100 \mathrm{~m}$, which is similar to the ceilometer. As expected, instantaneous comparison at zenith gives better accuracy results with a measurement difference up to $50 \mathrm{~m}$ (i.e., $\sigma_{h} / h \simeq$ $3 \%)$.

The results of the second validation case (altocumulus/multi-layer) show that all-sky camera network can identify multiple cloud layers. In this case, the offset between distribution peaks is $20 \mathrm{~m}$ for the lower cumulus fractus cloud layer $(h=1000 \mathrm{~m}$ a.g.l.). For the layer at $2300 \mathrm{~m}$ a.g.l., the offset on the distribution peak varies between $60 \mathrm{~m}$ (second image) and $350 \mathrm{~m}$ (three image). In this case, where $h / b=15$, the ratio $\sigma_{h} / h \simeq 15 \%$. As previously stated, standard deviations obtained by the cameras and the ceilometer are similar around the peak values, varying between 100 and $200 \mathrm{~m}$. For this case, instantaneous comparison at zenith gives a measurement difference up to $100 \mathrm{~m}$ for the $2300 \mathrm{~m}$ layer (i.e., $\sigma_{h} / h \simeq 5 \%$ ).

From these experiments, using peak distribution offsets, we note that $\sigma_{h} / h \simeq 0.01 h / b$ can be considered as a general rule for the height measurement uncertainty when using our methodology. The stereo calibration step is most likely responsible for the observed shifts. As we have explained in Sect. 2.3, this step is sensitive to the quality of the pixel matching performed by the SIFT method. This is illustrated by Fig. 12 showing variability of the height distribution with different stereo calibrations in the altocumulus case. According to $\sigma_{h} / h$ relationship, sensitivity on the stereo calibration step increases when ratio $h / b$ increases. Indeed, in this example, the peak corresponding to the low-layer cumulus fractus clouds $(\simeq 1000 \mathrm{~m})$ is barely impacted by the stereo calibration step.

\subsubsection{Horizontal georeferencing results, velocity map and segmentation}

The horizontal georeferencing and velocity results obtained for the cumulus and altocumulus/multi-layer cases are shown in Figs. 13 and 14, respectively. For each figure, we show the left camera rectified image and its associated velocity map (top figures), the 3-D point cloud projection on the left camera $x-y$ horizontal plane, and uncertainty on position $\sigma_{r}$ (bottom figures). This uncertainty is estimated using the Eq. (18) with $\sigma_{\phi}=2^{\circ}$, and $\sigma_{h}=0.01 h^{2} / b$ according to the experimental results presented in the previous section.

The results show the ability of the all-sky stereo system to retrieve information on cloud field spatial organization. As expected, position uncertainty increases with altitude and angle of incidence of the cloud. For the cumulus case, this uncertainty is about $120 \mathrm{~m}$ for a cloud located at a ground distance of $1 \mathrm{~km}, 250 \mathrm{~m}$ for a cloud located at $2 \mathrm{~km}$ and 

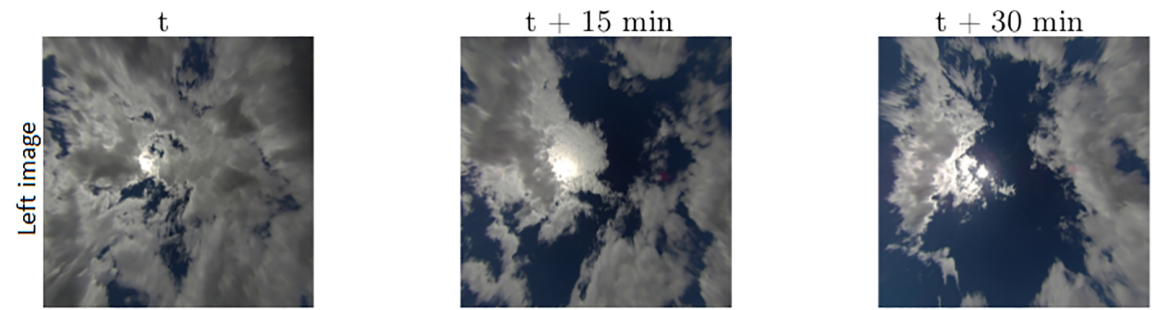

(a)
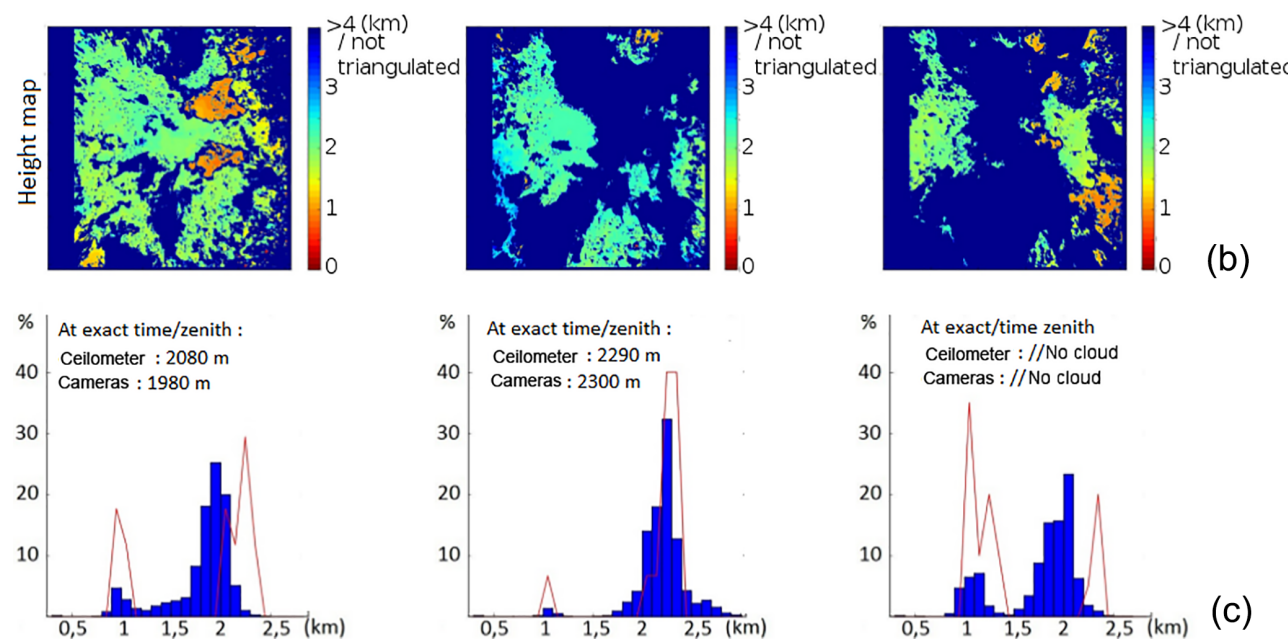

(c)

Figure 10. Altocumulus/multilayer validation case - height map and distribution for three shots evenly spaced of 15 min. Panel (a) represents the left camera images. Panel (b) represents the associated height map computed by the stereovision system. Panel (c) represents the frequency histogram of heights computed by the stereovision system (blue diagram - $100 \mathrm{~m}$ bins). This distribution is compared to the ceilometer frequency histogram (red curve - $100 \mathrm{~m}$ bins).

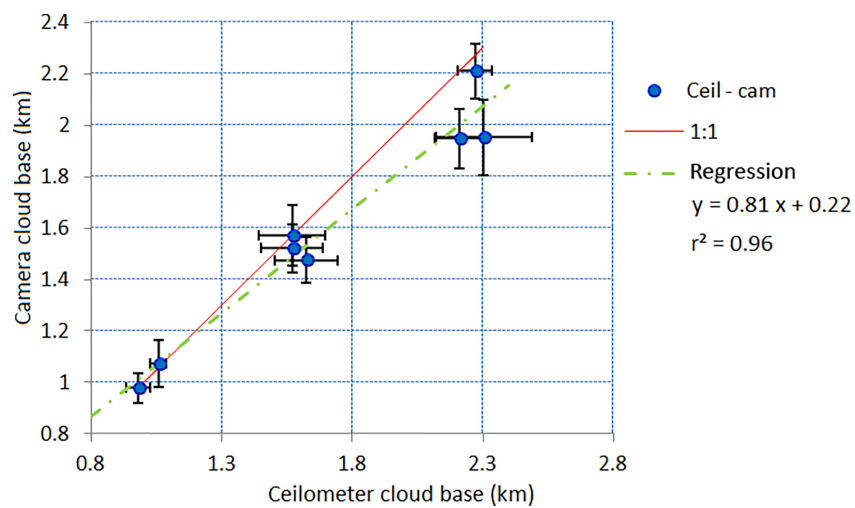

Figure 11. Comparison of mean cloud base height results obtained by the camera stereovision system and mean cloud base height results obtained by the ceilometer (blue points). The red $1: 1$ line corresponds to the reference plot. Linear regression for the ceilometercamera plot is shown by the green dashed line.

$450 \mathrm{~m}$ for a cloud located at $3 \mathrm{~km}$. The estimated velocity is $14 \mathrm{~km} \mathrm{~h}^{-1}$ with a mean direction of wind of $255^{\circ}$.

For the multi-layer/altocumulus case, the uncertainty is $180 \mathrm{~m}$ for a cloud at $1 \mathrm{~km}, 330 \mathrm{~m}$ for a cloud at $2 \mathrm{~km}$ and $500 \mathrm{~m}$ for a cloud at $3 \mathrm{~km}$. The velocity results show that the all-sky stereo system is able to estimate the velocities of dif- ferent cloud layers. In this case, the estimated average velocity is $16 \mathrm{~km} \mathrm{~h}^{-1}$ for the $1000 \mathrm{~m}$ layer and $30 \mathrm{~km} \mathrm{~h}^{-1}$ for the $2300 \mathrm{~m}$ layer, with respective mean directions of 205 and $230^{\circ}$.

We note that the uncertainty on cloud layer velocity is related to $h$ following Eq. (13), and is between 10 and $15 \%$ in the cases studied.

In the cumulus mediocris case, as we have separated cumulus clouds on the images, the situation allows going further and implementing the segmentation algorithm (Sect. 2.6). Results are shown in Fig. 15. We show the cloud height map, as well as the segmented image with the estimated positions of cloud centers (red dots and cloud identification number). The estimated cloud positions are listed in Table 4. The estimated individual cloud base heights are compared with the \pm 15 min ceilometer time series. In our case, we find that the all-sky camera network allows identifying clouds as individual objects. The estimated cloud base heights agree well with the ceilometer.

\section{Discussion and future work}

The results obtained under the configuration described in this study are relevant for macroscopically characterizing a cloud field up to $2500 \mathrm{~m}$ altitude, as well as cloud-targeting applica- 

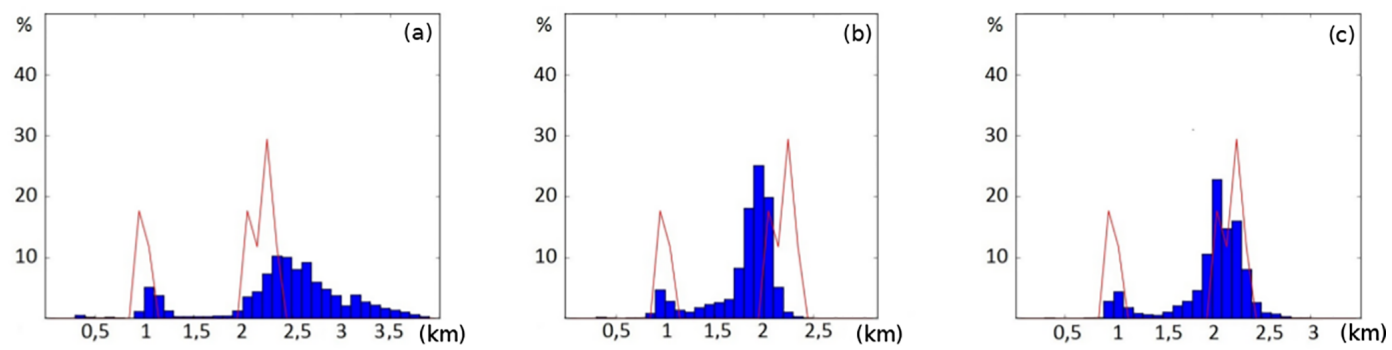

Figure 12. Sensitivity of the stereo calibration step illustrated by the first shot in the altocumulus validation case. For this shot, we represent the height-frequency histogram obtained with (a) no stereo calibration, (b) stereo calibration parameters obtained with the first shot pair of images, and (c) stereo calibration parameters obtained with the second shot pair of images.
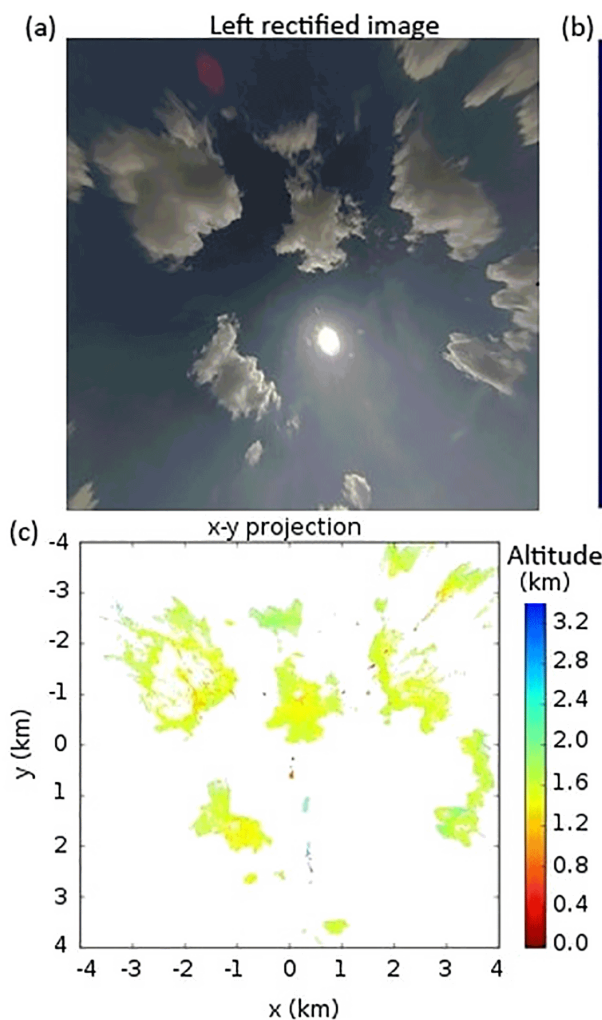

(b)

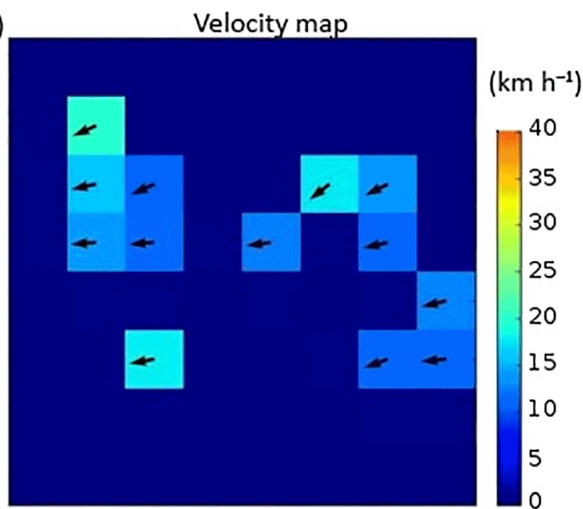

(d)

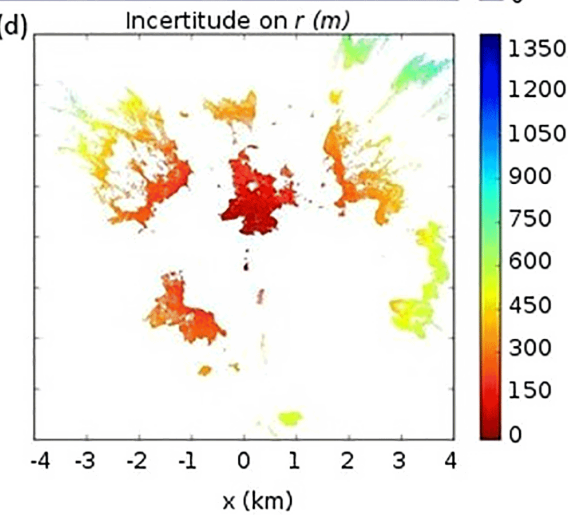

Figure 13. Cumulus case. (a, b) Rectified image (a) with estimated wind speed and direction (b). (c, d) Triangulated points projected on $x-y$ left camera plane with altitude color map (c), and with $r$-incertitude color map (d).

tions by instrumented UAVs. Yet, for precise measurements - morphological parameters of a cloud (width, vertical extension and variation over time), and precise geolocation (e.g., measurements near the base, top, or edges of the cloud) the all-sky camera network must be configured to ensure a certain accuracy.

In addition to optimizing the baseline distance between the cameras, several strategies can be explored to improve the accuracy of all-sky camera system. A first strategy is to work on the robustness of the orientation step. Relative orientation accuracy between stereo cameras plays an important role in the image rectification process (Sect. 2.3). Indeed, relative orientation has an impact on 3-D reconstruction accuracy through pixel-matching hit score, and uncertainty on disparity, as shown with Eqs. (12) and (15), and experimentally in Fig. 12. Moreover, it is important to ensure that cameras are correctly oriented in the Earth's frame for accurate geolocalization, as shown in Eq. (18).

In previous studies, the camera orientation is based on identified elements of the landscape, such as stars, trees, buildings and horizon lines. This consideration of external elements is adapted to the context of a fixed installation of a camera system, but becomes less suitable in the context of a measurement campaign in which the all-sky camera network 
Table 4. Segmentation and geolocalization results.

\begin{tabular}{lcrcc}
\hline Cloud ID & $\begin{array}{c}\text { Estimated cloud base } \\
\text { height (m a.g.1.) } \pm 10 \%\end{array}$ & $\begin{array}{r}\text { Position }(x, y) \text { of cloud centers } \\
\text { in the left rectified coordinate system }\end{array}$ & $r$ & $\sigma_{r}$ \\
\hline 3 & 1440 & $(-2.69 \mathrm{~km}, 1.75 \mathrm{~km})$ & $3.21 \mathrm{~km}$ & $\pm 350 \mathrm{~m}$ \\
5 & 1670 & $(2.41 \mathrm{~km}, 1.55 \mathrm{~km})$ & $2.87 \mathrm{~km}$ & $\pm 290 \mathrm{~m}$ \\
6 & 1420 & $(-1.83 \mathrm{~km}, 1.46 \mathrm{~km})$ & $2.34 \mathrm{~km}$ & $\pm 260 \mathrm{~m}$ \\
7 & 1450 & $(-1.80 \mathrm{~km},-0.23 \mathrm{~km})$ & $1.81 \mathrm{~km}$ & $\pm 170 \mathrm{~m}$ \\
9 & 1430 & $(1.35 \mathrm{~km},-1.57 \mathrm{~km})$ & $2.10 \mathrm{~km}$ & $\pm 210 \mathrm{~m}$ \\
10 & 1450 & $(-0.23 \mathrm{~km},-2.89 \mathrm{~km})$ & $2.90 \mathrm{~km}$ & $\pm 290 \mathrm{~m}$ \\
12 & 1640 & & & \\
\hline
\end{tabular}

Ceilometer cloud base heights measured during a 30 min time series: 1420-1450-1530-1350-1560-1550-1630-1620 m a.g.l.

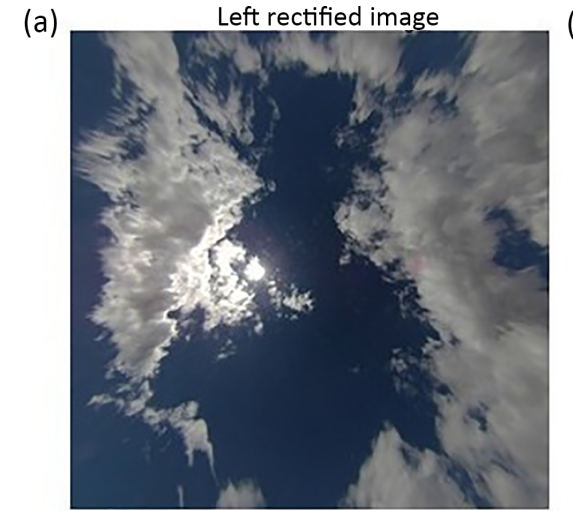

(c)

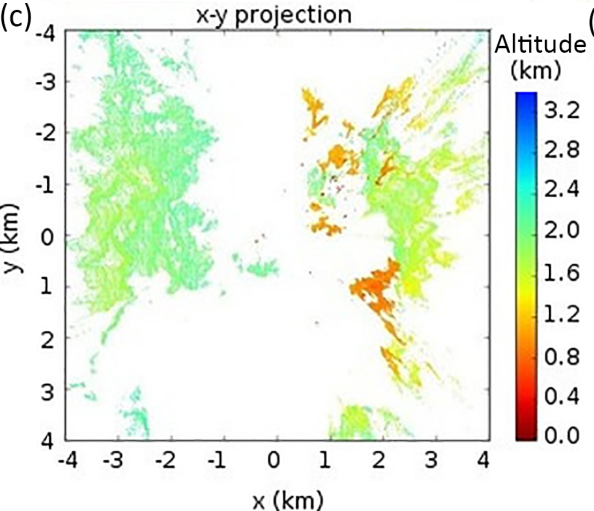

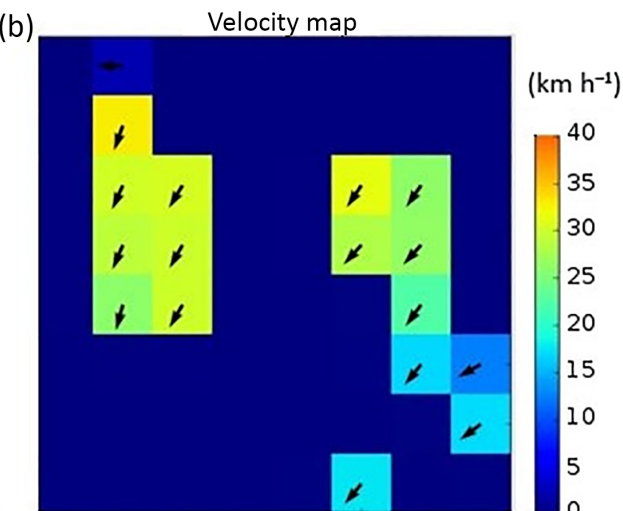

(d)

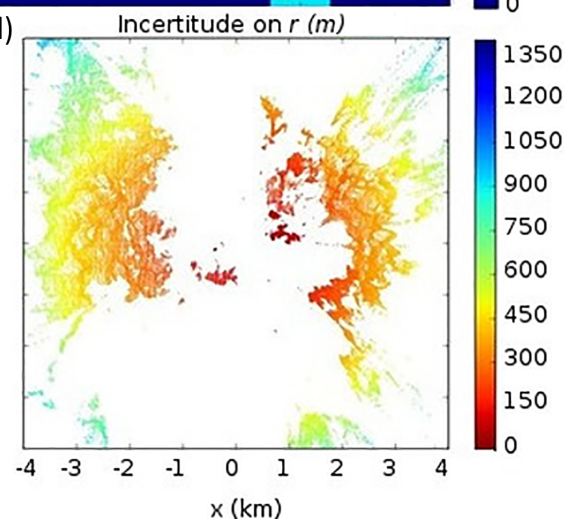

Figure 14. Altocumulus/multilayer case. (a, b) Rectified image (a) with estimated wind speed and direction (b). (c, d) Triangulated points projected on $x-y$ left camera plane with altitude color map (c), and with $r$-incertitude color map (d).

must be mobile and rapidly operational. The technique used here to initially orient the camera network is based on GPS for positioning in the Earth frame, leveling for horizontal adjustment, and vertical sights on the camera housing for intercamera alignment, which is a priori less accurate than using landmarks or stars to establish the orientation. Improving the initial orientation accuracy can be accomplished using laser sighting or the use of successive images of a GPS-equipped balloon or UAV loitering in the field of view of the cameras. In addition, the relative orientation between camera pairs can be refined by the stereo calibration algorithm using a time series of several pairs of images, instead of an instantaneous snapshot of a single pair of images. In addition, improved accuracy can also be achieved by organizing a network of several cameras (Heinrichs et al., 2007). For example, the arrangement of the cameras on the ground can be used to increase the number of triangulations of the same object (e.g., square arrangement with four cameras). Inter-camera spacing can also be organized to accommodate different cloud layers (e.g., closely spaced cameras for low clouds and farther apart for high-altitude clouds).

For dense stereo matching, the block-matching algorithm (Szeliski, 2010) yields correct results even in weakly textured areas, provided that smoothing and filtering techniques are 

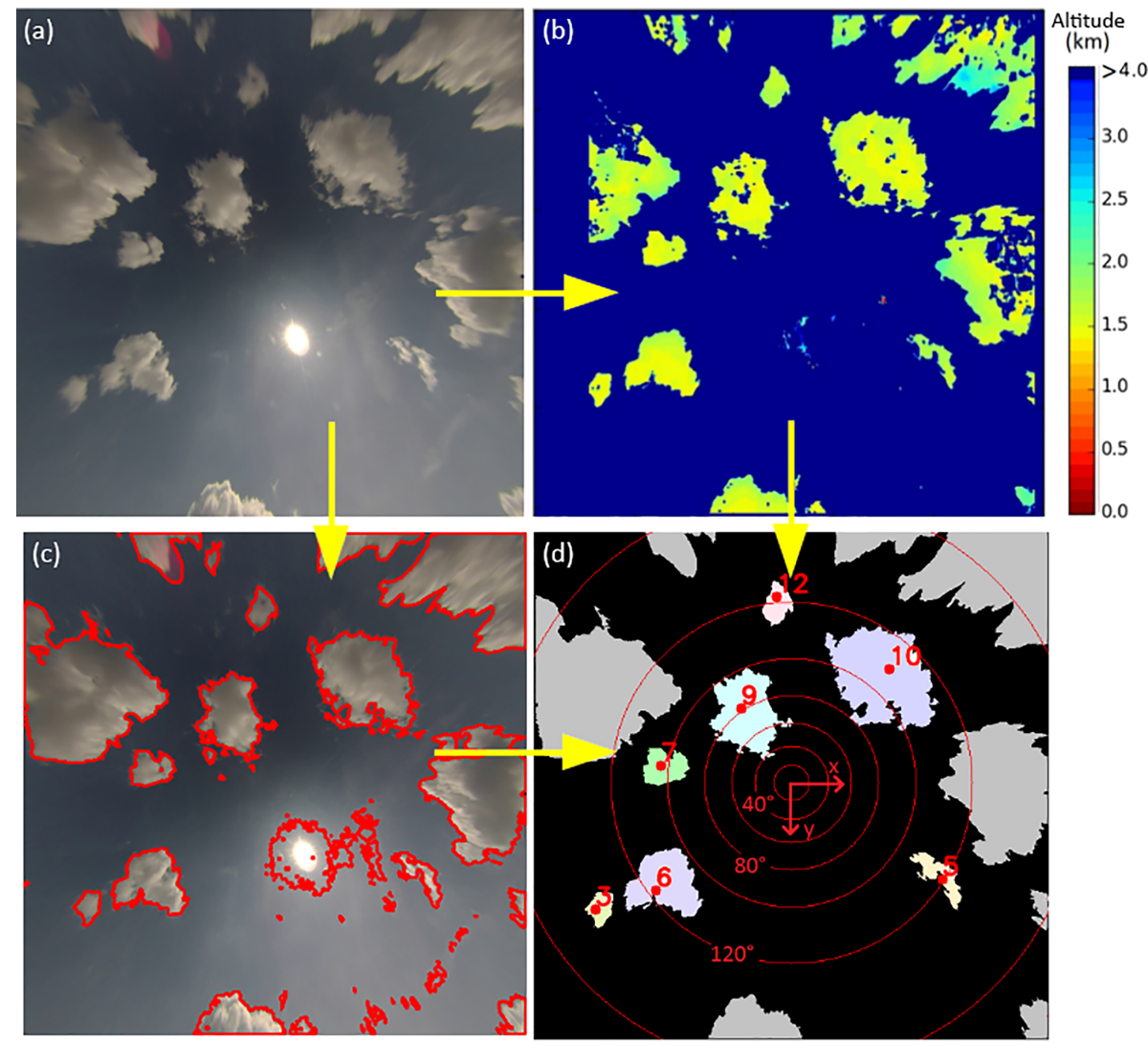

Figure 15. (a, b) Undistorted and rectified left image with associated height map. (c) Contours produced by blue filtering segmentation on left rectified image. (d) Segmented image with cloud identification number and estimated position of center of cloud base (red dots). Altitude filter: $4000 \mathrm{~m}$ a.g.l.

implemented (Sect. 2.4). However, smoothing step impacts accuracy when reconstructing cloud edges. Block-matching algorithm is a standard method and it would be useful to carry out a comparative study of the results given by dense matching methods developed recently. This field of research is very active and there is a dedicated benchmark online platform described in Scharstein and Szeliski (2002). One of the objectives of a future study would be to use this benchmark to identify and implement methods capable of accurately characterizing low-textured cloud zones, as well as edges.

In terms of image segmentation (e.g., identification of individual clouds) and geolocation, the methods and results presented in this article provide an overview of computer vision techniques to estimate individual cloud positions and their characteristics in a shallow cumulus cloud field. Segmentation based on contour detection of neighboring pixels makes it possible to isolate individual clouds. The cloud segmentation approach used in this study works well for distinguishable clouds on the image, but its performance is less reliable if this is not the case. The cloud segmentation method can be refined by taking into account the altitude map for more complex cloud fields where different clouds overlap on the image (e.g., multiple cloud layers, higher cloudiness, or deep convection). We see in Fig. 10 that the reconstruction algorithm determines low cumulus fractus edges within overlapping higher cloud layer. For a stratiform cloud layer with high cloudiness and less contrast, the segmentation approach would be modified to discern macroscopic differences in the cloud structure. Nonetheless, as mentioned in the previous paragraph, reconstructing accurate edges in situations where low-textured objects overlap remains a challenging task in the computer vision field. The uncertainty with respect to geo-localization of an individual cloud center position is directly related to uncertainty estimation on height (Sect. 2.7).

Finally, the use of photogrammetry techniques associated with segmentation opens the way to the characterization of other parameters of interest in atmospheric science, such as the width of the cloud base and the vertical extension of the cloud. The width of cloud base follows its growth and dissipation, and can be well estimated at low zenith angles. In contrast, extracting cloud vertical dimensions can be achieved at large zenith angles as long as the cloud tops are not hidden in the projection (Beekmans et al., 2016). Consequently, segmentation makes it possible to track individual clouds through successive images and follow the evolution 
of the cloud life cycle by tracking cloud heights and/or cloud base widths.

Data availability. No data sets were used in this article.

Competing interests. The authors declare that they have no conflict of interest.

Acknowledgements. This study was performed within the framework of the Skyscanner project supported by the STAE foundation and the Micro-Aerial Vehicle Research Center. We particularly thank Frédéric Murguet at Météo-France, and the CNRM/GMEI/MNPCA team for technical and organizational support. We also thank Samuel Lauda for preliminary studies on this project, and Simon Lacroix (CNRS/LAAS) for support on computer vision techniques.

Edited by: Mark Weber

Reviewed by: two anonymous referees

\section{References}

Allmen, M. C. and Kegelmeyer, J. P.: The computation of cloud base height from paired whole-sky imaging cameras, Mach. Vision Appl., 9, 160-165, 1997.

Beekmans, C., Schneider, J., Läbe, T., Lennefer, M., Stachniss, C., and Simmer, C.: Cloud photogrammetry with dense stereo for fisheye cameras, Atmos. Chem. Phys., 16, 14231-14248, https://doi.org/10.5194/acp-16-14231-2016, 2016.

Borque, P., Kollias, P., and Giangrande, S.: First observations of tracking clouds using scanning ARM cloud radars, J. Appl. Meteorol. Clim., 53, 2732-2746, 2014.

Bradbury, D. L. and Fujita, T. T.: Computation of height and velocity of clouds from dual, whole-sky, time-lapse picture sequences, University of Chicago, 1968.

Bradski, G. and Kaehler, A.: Learning OpenCV: Computer vision with the OpenCV library, O'Reilly Media, Inc., 2008.

Heinrichs, M., Rodehorst, V., and Hellwich, O.: Efficient semiglobal matching for trinocular stereo, differences (SSD), PIA07 Photogrammetric Image Analysis, 36, 185-190, 2007.

$\mathrm{Hu}$, J., Razdan, A., and Zehnder, J. A.: Geometric calibration of digital cameras for 3D cumulus cloud measurements, J. Atmos. Ocean. Tech., 26, 200-214, 2009.
IPCC: Climate Change 2013: The Physical Science Basis, Contribution of Working Group I to the Fifth Assessment Report of the Intergovernmental Panel on Climate Change, Cambridge University Press, Cambridge, UK, New York, NY, USA, 2013.

Janeiro, F. M., Carretas, F., Kandler, K., Wagner, F., and Ramos, P. M.: Advances in cloud base height and wind speed measurement through stereophotogrammetry with low cost consumer cameras, Measurement, 51, 429-440, 2014.

Koppe, C.: Photogrammetrie und internationale Wolkenmessung, F. Vieweg und Sohn, 108 pp., 1896.

Lewis, J. P.: Fast template matching, in: Vision interface, vol. 95, 15-19, 1995.

Lowe, D. G.: Distinctive image features from scale-invariant keypoints, Int. J. Comput. Vision, 60, 91-110, 2004.

Nguyen, D. A. and Kleissl, J.: Stereographic methods for cloud base height determination using two sky imagers, Sol. Energy, 107, 495-509, 2014.

Öktem, R., Lee, J., Thomas, A., Zuidema, P., and Romps, D. M.: Stereophotogrammetry of Oceanic Clouds, J. Atmos. Ocean. Tech., 31, 1482-1501, 2014.

Puig, L., Bermúdez, J., Sturm, P., and Guerrero, J. J.: Calibration of omnidirectional cameras in practice: A comparison of methods, Comput. Vis. Image Und., 116, 120-137, 2012.

Scaramuzza, D., Martinelli, A., and Siegwart, R.: A toolbox for easily calibrating omnidirectional cameras, in: 2006 IEEE/RSJ International Conference on Intelligent Robots and Systems, 56955701, 2006.

Scharstein, D. and Szeliski, R.: A taxonomy and evaluation of dense two-frame stereo correspondence algorithms, Int. J. Comput. Vision, 47, 7-42, 2002.

Seiz, G.: Ground-and satellite-based multi-view photogrammetric determination of 3D cloud geometry, $\mathrm{PhD}$ thesis, PennState University, USA, 2003.

Stevens, B. and Feingold, G.: Untangling aerosol effects on clouds and precipitation in a buffered system, Nature, 461, 607-613, 2009.

Suzuki, S. and Abe, K.: Topological structural analysis of digitized binary images by border following, Lect. Notes Comput. Sc., 30, 32-46, 1985.

Szeliski, R.: Computer vision: algorithms and applications, Springer Science \& Business Media, 957 pp., 2010.

Zhang, Z.: A flexible new technique for camera calibration, IEEE T. Pattern Anal., 22, 1330-1334, 2000. 